$\xi=\mathrm{\alpha}$

\title{
A 3-component mixture of inverse Rayleigh distributions: properties and estimation in Bayesian framework
}

\author{
Tabasam Sultana ${ }^{1}$, Muhammad Aslam ${ }^{2}$ \\ ${ }^{I}$ Department of Statistics, Quaid-i-Azam University, Islamabad, Pakistan \\ ${ }^{2}$ A Department of Basic Sciences, Ripha International University, Islamabad, 44000, Pakistan \\ *Corresponding author E-mail: tabasam_sultana@hotmail.com
}

\begin{abstract}
This paper is about studying a 3-component mixture of the inverse Rayleigh distributions under Bayesian perspective. The censored sampling scheme is considered due to its popularity in reliability theory and survival analysis. The expressions for the Bayes estimators and their posterior risks are derived under different loss scenarios. In case, no little prior information is available, elicitation of hyper parameters is given. To examine, numerically, the performance of the Bayes estimators using non-informative and informative priors under different loss functions, we have simulated their statistical properties for different sample sizes and test termination times.
\end{abstract}

Keywords: Bayes Estimators; Censoring, Loss Functions; Mixture Models; Posterior Risks.

\section{Introduction}

The inverse Rayleigh distribution has many applications in the area of reliability studies. Most of the lifetime distributions used in reliability studies is characterized by a monotone failure rate. However, one parameter inverse Rayleigh distribution has also been used as a failure time distribution Voda [22] mentioned that the distribution of lifetime of several types of experimental units can be approximated by the inverse Rayleigh distribution. Different studies have been used the inverse Rayleigh distribution for various purposes. For example, Gharraph [8] derived five measures of the parameter of inverse Rayleigh distribution and also obtained the estimators of the unknown parameter using different methods of estimation. Abdel-Monem [1] developed some estimation and prediction results for the inverse Rayleigh distribution. Soliman and Al-Aboud [20] used Bayesian and classical techniques for parameter estimation based on a set of upper record values from the Rayleigh distribution. Bayesian estimators have been developed under symmetric and asymmetric loss functions. Howlader et al. [10] used the Bayesian approach to predict the bounds for Rayleigh and inverse Rayleigh lifetime models. Soliman et al. [19] discussed the problems of Bayesian and nonBayesian estimation of an unknown parameter for an inverse Rayleigh distribution based on lower record values. Maximum likelihood estimate of the unknown parameter and Bayesian analysis was addressed using squared error and zero-one loss functions. The informative prior used to derive these estimates and the predictive intervals were also addressed with a real life data set. Dey [7] obtained Bayesian estimate of an inverse Rayleigh distribution using squared error and linear exponential loss functions.

The mixture models have established great interest for the analysts in the recent era. These models include finite and infinite number of components that can analyze different data sets. A finite mixture of some suitable probability distribution is recommended to study the population that is supposed to comprise a number of sub-populations mixing in an unknown proportion. Finite mixture models have been widely used in almost all fields of statistical sciences to model diverse populations. Fields in which mixture models have been successfully applied includes genetics, astronomy, medicine, engineering, economics; marketing etc. The analysis of mixture models under Bayesian framework has developed a significant interest among statisticians. Sultan et al. [21] discussed the properties of a 2-component mixture of the inverse Weibull distributions using a classical approach and the identifiably property of of the mixture model were also discussed. Kazmi et al. [14] described the Bayesian analysis for the 2-component mixture of Maxwell distributions. Noor and Aslam [17] studied Bayesian inference of the inverse Weibull mixture model using Type I censoring. Sajid Ali [2] described the 2-component mixture of the inverse Rayleigh distributions under Bayesian framework. Aslam and Tahir [4] presented the 3-component mixture of Rayleigh distribution under Bayesian framework.

Several types of data are encountered in everyday life, including simple data, grouped data, truncated data, censored data and progressively censored data. Censoring is an important and valuable aspect of the lifetime data. A valuable account of censoring is given in Gijbles [9] and Kalbfleisch and Prentice [13].

Motivated by above mentioned applications of mixture models, we plan to have Bayesian analysis of a 3-component mixture of the inverse Rayleigh distributions with unknown mixing proportions. The parameters of component distributions are assumed to be unknown. Four different priors and three different loss functions are used for the Bayesian analysis. In addition, we assume an ordinary Type I right censored sampling schemes.

The rest of the paper is organized as follows: The 3-component mixture of the inverse Rayleigh distributions is defined in section 2. The Likelihood function of the inverse Rayleigh mixture model is constructed in section 3. The expressions for posterior distributions using the non-informative and informative priors are derived in section 4. In section 5, the Bayes estimators and their posterior risks using the non-informative and informative priors under the squared error loss function (SELF), precautionary loss function (PLF) and DeGroot loss function (DLF) are presented. The elicita- 
tion of hyper parameters, if unknown is given in section 6. The limiting expression of the Bayes estimators and their posterior risks are derived in section 7 . The simulation study is presented in section 8. Finally, the conclusion of this study is given in section 9.

\section{Component mixture of the inverse Rayleigh distributions}

The probability density function (p.d.f) and the cumulative distribution function (c.d.f) of the inverse Rayleigh distribution for a random variable $\mathrm{X}$ are given by:

$$
\begin{aligned}
& f_{m}\left(x ; \theta_{m}\right)=\frac{2 \theta_{m}}{x^{3}} \exp \left(\frac{-\theta_{m}}{x^{2}}\right) ; x \geq 0, \theta_{m}>0, m=1,2,3 \\
& F_{m}(x)=\exp \left(\frac{-\theta_{m}}{x^{2}}\right) ; m=1,2,3
\end{aligned}
$$

Where $\theta_{\mathrm{m}}$ is the parameter of the inverse Rayleigh distribution.

A finite 3-component mixture model with the unknown mixing proportions $\mathrm{p}_{1}$ and $\mathrm{p}_{2}$ is defined as

$$
\begin{aligned}
& \mathrm{f}(\mathrm{x})=\mathrm{p}_{1} \mathrm{f}_{1}(\mathrm{x})+\mathrm{p}_{2} \mathrm{f}_{2}(\mathrm{x})+\left(1-\mathrm{p}_{1}-\mathrm{p}_{2}\right) \mathrm{f}_{3}(\mathrm{x}), \\
& \mathrm{p}_{1}, \mathrm{p}_{2} \geq 0, \mathrm{p}_{1}+\mathrm{p}_{2} \leq 1 \\
& \mathrm{f}\left(\mathrm{x}, \theta_{1}, \theta_{2}, \theta_{3}, \mathrm{p}_{1}, \mathrm{p}_{2}\right)=\mathrm{p}_{1} \frac{2 \theta_{1}}{\mathrm{x}^{3}} \exp \left(\frac{-\theta_{1}}{\mathrm{x}^{2}}\right)+\mathrm{p}_{2} \frac{2 \theta_{2}}{\mathrm{x}^{3}} \exp \left(\frac{-\theta_{2}}{\mathrm{x}^{2}}\right) \\
& +\left(1-\mathrm{p}_{1}-\mathrm{p}_{2}\right) \frac{2 \theta_{3}}{\mathrm{x}^{3}} \exp \left(\frac{-\theta_{3}}{\mathrm{x}^{2}}\right)
\end{aligned}
$$

While the c.d.f of the 3-component mixture of the Inverse Rayleigh distribution is given by:

$$
\begin{aligned}
& F(x)=p_{1} F_{1}(x)+p_{2} F_{2}(x)+\left(1-p_{1}-p_{2}\right) F_{3}(x) \\
& F(x)=p_{1} \exp \left(\frac{-\theta_{1}}{x^{2}}\right)+p_{2} \exp \left(\frac{-\theta_{2}}{x^{2}}\right)+\left(1-p_{1}-p_{2}\right) \exp \left(\frac{-\theta_{3}}{x^{2}}\right)
\end{aligned}
$$

\section{The likelihood function}

Suppose ' $n$ ' units from the 3-component mixture of inverse Rayleigh distributions are used in a life testing experiment with fixed test termination time $t$. Let ' $r$ ' units out of ' $n$ ' units failed until fixed test termination time ' $t$ ' and the remaining (n-r) units are still working. According to Mendenhall and Hader [16], there are many practical situations in which the failed objects can be pointed out easily as subset of subpopulation-1, subpopulation-II or subpopulation-III. Out of ' $r$ ' units, suppose $\mathrm{r}_{1}, \mathrm{r}_{2}$ and $\mathrm{r}_{3}$ units belong to subpopulation-1, subpopulation-II or subpopulation-III respectively and such that $r=r_{1}+r_{2}+r_{3}$. Now we define $x_{l k}$, $0<\mathrm{x}_{\mathrm{lk}}<\mathrm{t}$ be the failure time of $\mathrm{k}^{\text {th }}$ unit belonging to the $l^{\text {th }}$ subpopulation, where $l=1,2,3$ and $k=1,2, \ldots, r_{l}$. For a 3-component mixture model, the likelihood function can be written as

$$
\begin{aligned}
& \mathrm{L}\langle\varphi \mid \mathrm{x}\rangle \propto\left\{\prod_{\mathrm{k}=1}^{\mathrm{r}_{1}} \mathrm{p}_{1} \mathrm{f}_{1}\left(\mathrm{x}_{1 \mathrm{k}}\right)\right\}\left\{\prod_{\mathrm{k}=1}^{\mathrm{r}} \mathrm{p}_{2} \mathrm{f}_{2}\left(\mathrm{x}_{2 \mathrm{k}}\right)\right\} \\
& \left\{\begin{array}{l}
\left.\mathrm{r}_{3}\left(1-\mathrm{p}_{1}-\mathrm{p}_{2}\right) \mathrm{f}_{3}\left(\mathrm{x}_{3 \mathrm{k}}\right)\right\} \\
\mathrm{k}=1
\end{array}\right\}[1-\mathrm{F}(\mathrm{t})]^{\mathrm{n}-\mathrm{r}}
\end{aligned}
$$

After simplification, the likelihood function of 3-component mixture of Inverse Rayleigh distribution is given by:

$$
\begin{aligned}
& \mathrm{L}\langle\varphi \mid \mathrm{x}\rangle \propto \theta_{1}^{\mathrm{r}_{1}} \theta_{2}^{\mathrm{r}_{2}} \theta_{3}^{\mathrm{r}_{3}} \sum_{\mathrm{i}=0}^{\mathrm{n}-\mathrm{r}} \sum_{\mathrm{j}=0}^{\mathrm{i}} \sum_{1=0}^{\mathrm{j}}(-1)^{\mathrm{i}}\left(\begin{array}{l}
\mathrm{n}-\mathrm{r} \\
\mathrm{i}
\end{array}\right)\left(\begin{array}{l}
\mathrm{i} \\
\mathrm{j}
\end{array}\right)\left(\begin{array}{l}
\mathrm{j} \\
1
\end{array}\right) \\
& \exp \left\{-\theta_{1}\left(\sum_{\mathrm{k}=1}^{\mathrm{r}_{\mathrm{i}}} \mathrm{x}_{1 \mathrm{k}}^{-2}+\frac{\mathrm{i}-\mathrm{j}}{\mathrm{t}^{2}}\right)\right\} \\
& \exp \left\{-\theta_{2}\left(\sum_{\mathrm{k}=1}^{\mathrm{r}_{2}} \mathrm{x}_{2 \mathrm{k}}^{-2}+\frac{\mathrm{j}-1}{\mathrm{t}^{2}}\right)\right\}
\end{aligned}
$$

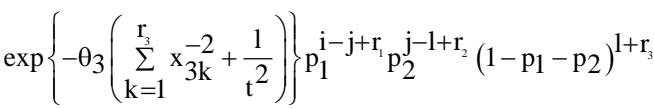

Where

$\varphi=\left(\theta_{1}, \theta_{2}, \theta_{3}, p_{1}, p_{2}\right)$ and $X=\left(x_{11}, \ldots, x_{1 r_{1}}, x_{21}, \ldots, x_{2 r_{2}}, x_{31}, \ldots, x_{3 r_{3}}\right)$

Are the observed failure times for the uncensored observations

\section{The posterior distribution using the non- informative and the informative priors}

In this section, posterior distributions of parameters given data, say $\mathrm{x}$, are derived using the non-informative (Uniform and Jeffreys') and the informative (Gamma and Exponential) priors.

\subsection{The posterior distribution using the uniform prior (UP)}

When elicitation of hyper parameters is difficult or little prior information is given, then usually the non-informative prior is assumed to be the UP. Ups over the intervals $(0, \infty)$ and $(0,1)$ are taken for the parameters $\left(\theta_{1}, \theta_{2} \& \theta_{3}\right)$ of Inverse Rayleigh distribution and for the mixing proportions $\left(\mathrm{p}_{1}, \mathrm{p}_{2}\right)$ respectively. With these settings, joint prior distribution of parameters $\left(\theta_{1}, \theta_{2}, \theta_{3}, p_{1}, p_{2}\right)$, is given by:

$\pi_{1}(\varphi) \propto 1 ; \theta_{1}, \theta_{2}, \theta_{3}>0, p_{1}, p_{2} \geq 0, p_{1}+p_{2} \leq 1$

The joint posterior distribution of parameters $\theta_{1}, \theta_{2}, \theta_{3}, \mathrm{p}_{1}$ and $\mathrm{p}_{2}$ given data $\mathrm{x}$ assuming the UP is:

$$
\begin{aligned}
& \mathrm{g}_{1}\langle\varphi \mid \mathrm{x}\rangle=\frac{\mathrm{L}\langle\varphi \mid \mathrm{x}\rangle \pi_{1}(\varphi)}{\int \mathrm{L}\langle\varphi \mid \mathrm{x}\rangle \pi_{1}(\varphi) \mathrm{d} \varphi} \\
& \varphi \\
& \mathrm{g}_{1}\langle\varphi \mid \mathrm{x}\rangle=\Omega_{1}^{-1} \sum_{\mathrm{i}=0}^{\mathrm{n}-\mathrm{r}} \sum_{\mathrm{j}=01=0}^{\mathrm{i}} \sum_{1=0}^{\mathrm{j}}(-1)^{\mathrm{i}}\left(\begin{array}{l}
\mathrm{n}-\mathrm{r} \\
\mathrm{i}
\end{array}\right)\left(\begin{array}{l}
\mathrm{i} \\
\mathrm{j}
\end{array}\right)\left(\begin{array}{l}
\mathrm{j} \\
1
\end{array}\right) \theta_{1}^{\mathrm{A}_{11}-1} \theta_{2}^{\mathrm{A}_{21}-1} \theta_{3}^{\mathrm{A}_{31}-1} \\
& \exp \left(-\theta_{1} \mathrm{~B}_{11}\right) \exp \left(-\theta_{2} \mathrm{~B}_{21}\right) \\
& \exp \left(-\theta_{3} \mathrm{~B}_{31}\right) \mathrm{p}_{1}^{\mathrm{A}_{01}-\mathrm{p}_{2} \mathrm{~B}_{01}-1}\left(1-\mathrm{p}_{1}-\mathrm{p}_{2}\right)^{\mathrm{C}_{01}-1}
\end{aligned}
$$

Where

$$
\begin{aligned}
& A_{11}=r_{1}+1, A_{21}=r_{2}+1, A_{31}=r_{3}+1, B_{11} \\
& =\sum_{k=1}^{r_{1}} x_{1 k}^{-2}+\frac{i-j}{t^{2}}, B_{21}=\sum_{k=1}^{r_{2}} x_{2 k}^{-2}+\frac{j-1}{t^{2}}, \\
& B_{31}=\sum_{k=1}^{r_{3}} x_{3 k}^{-2}+\frac{1}{t^{2}}, A_{01}=i-j+r_{1}+1, B_{01} \\
& =j-1+r_{2}+1, C_{01}=1+r_{3}+1
\end{aligned}
$$




$$
\begin{aligned}
& \Omega_{1}=\sum_{\mathrm{i}=0}^{\mathrm{n}-\mathrm{r}} \sum_{\mathrm{j}=01}^{\mathrm{i}} \sum_{1=0}^{\mathrm{j}}(-1)^{\mathrm{i}}\left(\begin{array}{l}
\mathrm{n}-\mathrm{r} \\
\mathrm{i}
\end{array}\right)\left(\begin{array}{l}
\mathrm{i} \\
\mathrm{j}
\end{array}\right)\left(\begin{array}{l}
\mathrm{j} \\
1
\end{array}\right) \mathrm{B}\left(\mathrm{A}_{01}, \mathrm{C}_{01}\right) \\
& \mathrm{B}\left(\mathrm{B}_{01}, \mathrm{~A}_{01}+\mathrm{C}_{01}\right) \frac{\Gamma\left(\mathrm{A}_{11}\right)}{\mathrm{B}_{11}^{\mathrm{A}_{11}}} \frac{\Gamma\left(\mathrm{A}_{21}\right)}{\mathrm{B}_{21}^{\mathrm{A}_{21}}} \frac{\Gamma\left(\mathrm{A}_{31}\right)}{\mathrm{B}_{31}^{\mathrm{A}_{31}}}
\end{aligned}
$$

\subsection{The posterior distribution using the Jeffreys' prior (JP)}

According to Jeffreys' [11], [12] and Berger [5], the JP is defined as

$$
\mathrm{p}\left(\theta_{\mathrm{m}}\right) \propto \sqrt{\mathrm{I}\left(\theta_{\mathrm{m}}\right) \mid}, \mathrm{m}=1,2,3 \text {, where } \mathrm{I}\left(\theta_{\mathrm{m}}\right)=-\mathrm{E}\left[\frac{\partial^{2} \mathrm{f}\left\langle\mathrm{x} \mid \theta_{\mathrm{m}}\right\rangle}{\partial \theta_{\mathrm{m}}^{2}}\right]
$$

is the Fisher's information matrix. The prior distributions of the mixing proportions $\mathrm{p}_{1}$ and $\mathrm{p}_{2}$ are again taken to be the uniform over the interval $(0,1)$. Under the assumption of independence of all parameters, the joint prior distribution of $\left(\theta_{1}, \theta_{2}, \theta_{3}, \mathrm{p}_{1}, \mathrm{p}_{2}\right)$ is:

$$
\pi_{2}(\varphi) \propto \frac{1}{\theta_{1} \theta_{2} \theta_{3}}, \theta_{1}, \theta_{2}, \theta_{3} \geq 0, \mathrm{p}_{1}, \mathrm{p}_{2} \geq 0, \mathrm{p}_{1}+\mathrm{p}_{2} \leq 1
$$

The joint posterior distribution of parameters $\theta_{1}, \theta_{2}, \theta_{3}, \mathrm{p}_{1}$ and $\mathrm{p}_{2}$ given data $\mathrm{x}$ assuming the JP is:

$$
\begin{aligned}
& \mathrm{g}_{2}\langle\varphi \mid \mathrm{x}\rangle=\frac{\mathrm{L}\langle\varphi \mid \mathrm{x}\rangle \pi_{2}(\varphi)}{\int_{\varphi}^{\mathrm{L}\langle\varphi \mid \mathrm{x}\rangle \pi_{2}(\varphi) \mathrm{d} \varphi}} \\
& \mathrm{g}_{2}\langle\varphi \mid \mathrm{x}\rangle= \\
& \Omega_{2}^{-1} \sum_{\mathrm{i}=0}^{\mathrm{n}-\mathrm{r}} \sum_{\mathrm{j}=01}^{\mathrm{i}} \sum_{1=0}^{\mathrm{j}}(-1)^{\mathrm{i}}\left(\begin{array}{l}
\mathrm{n}-\mathrm{r} \\
\mathrm{i}
\end{array}\right)\left(\begin{array}{l}
\mathrm{i} \\
\mathrm{j}
\end{array}\right)\left(\begin{array}{l}
\mathrm{j} \\
1
\end{array}\right) \theta_{1}^{\mathrm{A}_{12}-1} \theta_{2}^{\mathrm{A}_{22}-1_{\theta_{3}} \mathrm{~A}_{32}-1} \\
& \exp \left(-\theta_{1} \mathrm{~B}_{12}\right) \\
& \exp \left(-\theta_{2} \mathrm{~B}_{22}\right) \\
& \exp \left(-\theta_{3} \mathrm{~B}_{32}\right) \mathrm{p}_{1}^{\mathrm{A}_{02}-1} \mathrm{p}_{2}^{\mathrm{B}_{02}-1}\left(1-\mathrm{p}_{1}-\mathrm{p}_{2}\right)^{\mathrm{C}_{02}-1}
\end{aligned}
$$

Where

$$
\begin{aligned}
& A_{12}=r_{1}, A_{22}=r_{2}, A_{32}=r_{3}, B_{12} \\
& =\sum_{k=1}^{r_{1}} x_{1 k}^{-2}+\frac{i-j}{t^{2}}, B_{22}=\sum_{k=1}^{r_{2}} x_{2 k}^{-2}+\frac{j-1}{t^{2}}, \\
& B_{32}=\sum_{k=1}^{r_{3}} x_{3 k}^{-2}+\frac{1}{t^{2}}, A_{02}=i-j+r_{1}+1, B_{02} \\
& =j-1+r_{2}+1, C_{02}=1+r_{3}+1, \\
& \Omega_{2}=\sum_{i=0}^{n-r} \sum_{j=01}^{i} \sum_{1=0}^{j}(-1)\left(\begin{array}{l}
n-r \\
i
\end{array}\right)\left(\begin{array}{l}
i \\
j
\end{array}\right)\left(\begin{array}{l}
j \\
1
\end{array}\right) B\left(A_{02}, C_{02}\right) \\
& B\left(B_{02}, A_{02}+C_{02}\right) \frac{\Gamma\left(A_{12}\right)}{B_{12}^{A_{12}}} \frac{\Gamma\left(A_{22}\right)}{B_{22}^{A_{22}}} \frac{\Gamma\left(A_{32}\right)}{B_{32}^{A_{32}}}
\end{aligned}
$$

\subsection{The posterior distribution using the gamma prior (GP)}

As an informative prior, we take the Gamma prior for the component parameters $\theta_{1}, \theta_{2}, \theta_{3}$ and bivariate beta prior for proportion parameters $\mathrm{p}_{1}, \mathrm{p}_{2}$.Symbolically, it can be written as: $\theta_{1} \sim \operatorname{Gamma}\left(a_{1}, b_{1}\right), \theta_{2} \sim \operatorname{Gamma}\left(a_{2}, b_{2}\right), \theta_{3} \sim \operatorname{Gamma}\left(a_{3}, b_{3}\right) \quad$ and $\mathrm{p}_{1}, \mathrm{p}_{2} \sim$ Bivariate Beta $(\mathrm{a}, \mathrm{b}, \mathrm{c})$.Again assuming independence of all parameters, the joint prior distribution of $\left(\theta_{1}, \theta_{2}, \theta_{3}, \mathrm{p}_{1}, \mathrm{p}_{2}\right)$ is given by:

$$
\begin{aligned}
& \pi_{3}(\varphi) \propto \theta_{1}^{a_{1}-1} \exp \left(-b_{1} \theta_{1}\right) \theta_{2}^{a_{2}-1} \exp \left(-b_{2} \theta_{2}\right) \theta_{3}^{a_{3}-1} \\
& \exp \left(-b_{3} \theta_{3}\right) p_{1}^{a-1} p_{2}^{b-1}\left(1-p_{1}-p_{2}\right)^{c-1}
\end{aligned}
$$

The joint posterior distribution of parameters $\theta_{1}, \theta_{2}, \theta_{3}, \mathrm{p}_{1}$ and $\mathrm{p}_{2}$ given data $\mathrm{x}$

$$
\mathrm{g}_{3}\langle\varphi \mid \mathrm{x}\rangle=\frac{\mathrm{L}\langle\varphi \mid \mathrm{x}\rangle \pi_{3}(\varphi)}{\int_{\varphi} \mathrm{L}\langle\varphi \mid \mathrm{x}\rangle \pi_{3}(\varphi) \mathrm{d} \varphi}
$$

$$
\begin{aligned}
& \mathrm{g}_{3}\langle\varphi \mid \mathrm{x}\rangle=\Omega_{3}^{-1} \sum_{\mathrm{i}=0}^{\mathrm{n}-\mathrm{r}} \sum_{\mathrm{j}=01}^{\mathrm{i}} \sum_{1=0}^{\mathrm{j}}(-1)^{\mathrm{i}}\left(\begin{array}{l}
\mathrm{n}-\mathrm{r} \\
\mathrm{i}
\end{array}\right)\left(\begin{array}{l}
\mathrm{i} \\
\mathrm{j}
\end{array}\right)\left(\begin{array}{l}
\mathrm{j} \\
1
\end{array}\right) \theta_{1}^{\mathrm{A}_{13}-1} \theta_{2}^{\mathrm{A}_{23}-1} \theta_{3}^{\mathrm{A} 3-1} \\
& \exp \left(-\theta_{1} \mathrm{~B}_{13}\right) \exp \left(-\theta_{2} \mathrm{~B}_{23}\right) \\
& \exp \left(-\theta_{3} \mathrm{~B}_{33}\right) \mathrm{p}_{1}^{\mathrm{A}_{03}-1} \mathrm{p}_{2}^{\mathrm{B}_{03}-1}\left(1-\mathrm{p}_{1}-\mathrm{p}_{2}\right)^{\mathrm{C}_{03}-1}
\end{aligned}
$$

Where

$A_{13}=r_{1}+a_{1}, A_{23}=r_{2}+a_{2}, A_{33}=r_{3}+a_{3}, B_{13}$

$=\sum_{k=1}^{r_{1}} x_{1 k}^{-2}+\frac{i-j}{t^{2}}+b_{1}, B_{23}=\sum_{k=1}^{r_{2}} x_{2 k}^{-2}+\frac{j-1}{t^{2}}+b_{2}$,

$\mathrm{B}_{33}=\sum_{\mathrm{k}=1}^{\mathrm{r}_{3}} \mathrm{x}_{3 \mathrm{k}}^{-2}+\frac{1}{\mathrm{t}^{2}}+\mathrm{b}_{3}, \mathrm{~A}_{03}=\mathrm{i}-\mathrm{j}+\mathrm{r}_{1}+\mathrm{a}, \mathrm{B}_{03}$

$=\mathrm{j}-1+\mathrm{r}_{2}+\mathrm{b}, \mathrm{C}_{03}=1+\mathrm{r}_{3}+\mathrm{c}$,

$\Omega_{3}=\sum_{i=0}^{n-r} \sum_{j=01=0}^{i} \sum_{1}^{j}(-1)^{i}\left(\begin{array}{l}n-r \\ i\end{array}\right)\left(\begin{array}{l}i \\ j\end{array}\right)\left(\begin{array}{l}j \\ 1\end{array}\right) B\left(A_{03}, C_{03}\right)$

$\mathrm{B}\left(\mathrm{B}_{03}, \mathrm{~A}_{03}+\mathrm{C}_{03}\right) \frac{\Gamma\left(\mathrm{A}_{13}\right)}{\mathrm{B}_{13}^{\mathrm{A}_{13}}} \frac{\Gamma\left(\mathrm{A}_{23}\right)}{\mathrm{B}_{23}^{\mathrm{A}_{23}}} \frac{\Gamma\left(\mathrm{A}_{33}\right)}{\mathrm{B}_{33}^{\mathrm{A}_{33}}}$

\subsection{The posterior distribution using the exponential prior (EP)}

As an informative prior, we take the Exponential prior for the component parameters $\theta_{1}, \theta_{2}, \theta_{3}$ and bivariate beta prior for proportion parameters $\mathrm{p}_{1}, \mathrm{p}_{2}$.Symbolically, it can be written $\theta_{1} \sim \operatorname{Exponential}\left(w_{1}\right), \theta_{2} \sim \operatorname{Exponential}\left(w_{2}\right), \theta_{3} \sim \operatorname{Exponential}\left(w_{3}\right)$ and $\mathrm{p}_{1}, \mathrm{p}_{2} \sim$ Bivariate Beta $(\mathrm{a}, \mathrm{b}, \mathrm{c})$.Again assuming independence of all parameters, the joint prior distribution of $\left(\theta_{1}, \theta_{2}, \theta_{3}, \mathrm{p}_{1}, \mathrm{p}_{2}\right)$ is given by:

$$
\begin{aligned}
& \pi_{4}(\varphi) \propto \mathrm{w}_{1} \exp \left(-\mathrm{w}_{1} \theta_{1}\right) \mathrm{w}_{2} \exp \left(-\mathrm{w}_{2} \theta_{2}\right) \\
& \mathrm{w}_{3} \exp \left(-\mathrm{w}_{3} \theta_{3}\right) \mathrm{p}_{1}^{\mathrm{a}-1} \mathrm{p}_{2}^{\mathrm{b}-1}\left(1-\mathrm{p}_{1}-\mathrm{p}_{2}\right)^{\mathrm{c}-1}
\end{aligned}
$$

The joint posterior distribution of parameters $\theta_{1}, \theta_{2}, \theta_{3}, \mathrm{p}_{1}$ and $\mathrm{p}_{2}$ given data $\mathrm{x}$

$$
\mathrm{g}_{4}\langle\varphi \mid \mathrm{x}\rangle=\frac{\mathrm{L}\langle\varphi \mid \mathrm{x}\rangle \pi_{4}(\varphi)}{\int \mathrm{L}\langle\varphi \mid \mathrm{x}\rangle \pi_{4}(\varphi) \mathrm{d} \varphi}
$$




$$
\begin{aligned}
& \mathrm{g}_{4}\langle\varphi \mid \mathrm{x}\rangle \\
& =\Omega_{4}^{-1} \sum_{\mathrm{i}=0}^{\mathrm{n}-\mathrm{r}} \sum_{\mathrm{j}=01=0}^{\mathrm{i}} \sum_{1}^{\mathrm{j}}(-1)^{\mathrm{i}}\left(\begin{array}{l}
\mathrm{n}-\mathrm{r} \\
\mathrm{i}
\end{array}\right)\left(\begin{array}{l}
\mathrm{i} \\
\mathrm{j}
\end{array}\right)\left(\begin{array}{l}
\mathrm{j} \\
1
\end{array}\right) \theta_{1}^{\mathrm{A}_{14}-1} \theta_{2}^{\mathrm{A}_{24}-1} \theta_{3}^{\mathrm{A}_{34}-1} \\
& \exp \left(-\theta_{1} \mathrm{~B}_{14}\right) \\
& \exp \left(-\theta_{2} \mathrm{~B}_{24}\right) \exp \left(-\theta_{3} \mathrm{~B}_{34}\right) \mathrm{p}_{1}^{\mathrm{A}_{04}-1} \mathrm{p}_{2}^{\mathrm{B}_{04}-1}\left(1-\mathrm{p}_{1}-\mathrm{p}_{2}\right)^{\mathrm{C}_{04}-1}
\end{aligned}
$$

Where

$$
\begin{aligned}
& A_{14}=r_{1}+1, A_{24}=r_{2}+1, A_{34}=r_{3}+1, B_{14} \\
& =\sum_{k=1}^{r_{1}} x_{1 k}^{-2}+\frac{i-j}{t^{2}}+w_{1}, B_{24}=\sum_{k=1}^{r_{2}} x_{2 k}^{-2}+\frac{j-1}{t^{2}}+w_{2}, \\
& B_{34}=\sum_{k=1}^{r_{3}} x_{3 k}^{-2}+\frac{1}{t^{2}}+w_{3}, A_{04}=i-j+r_{1}+a, B_{04} \\
& =j-1+r_{2}+b, C_{04}=1+r_{3}+c, \\
& \Omega_{4}=\sum_{i=0}^{n-r} \sum_{j=01}^{i} \sum_{j}^{j}(-1){ }^{i}\left(\begin{array}{l}
n-r \\
i
\end{array}\right)\left(\begin{array}{l}
i \\
j
\end{array}\right)\left(\begin{array}{l}
j \\
1
\end{array}\right) B\left(A_{04}, C_{04}\right) \\
& B\left(B_{04}, A_{04}+C_{04}\right) \frac{\Gamma\left(A_{14}\right)}{B_{14}^{A_{14}}} \frac{\Gamma\left(A_{24}\right)}{B_{24}^{A_{24}}} \frac{\Gamma\left(A_{34}\right)}{B_{34}^{A_{34}}}
\end{aligned}
$$

\section{Bayes estimators and posterior risks using the UP, the JP, the gamma and the expo- nential prior under SELF, PLF and DLF}

If $\hat{\mathrm{d}}$ is a Bayes estimator then $\rho(\hat{\mathrm{d}})$ is called posterior risk and is defined as: $\rho(\hat{\mathrm{d}})=\mathrm{E}_{\theta \mid \mathrm{x}}\{\mathrm{L}(\theta, \hat{\mathrm{d}})\}$. Our purpose, in this study, is to look for efficient Bayes estimators of the different parameters. For this purpose, three different loss functions, namely SELF, PLF and DLF used to obtain Bayes estimators and their posterior risks. The SELF, defined as $L(\theta, d)=(\theta-d)^{2}$, was introduced by Legendre [15] to develop the least squares theory. Norstrom [18] discussed an asymmetric PLF and also introduced a special case of general class of PLFs, which is defined as $L(\theta, d)=\frac{(\theta-d)^{2}}{d}$. While the DLF is presented by DeGroot [6] and is defined as $\mathrm{L}(\theta, \mathrm{d})=\left(\frac{\theta-\mathrm{d}}{\mathrm{d}}\right)^{2}$.

For a given prior, the Bayes estimator and posterior risk under SELF are calculated as: $\hat{\mathrm{d}}=\mathrm{E}_{\theta \mid \mathrm{x}}(\theta)$ and $\rho(\hat{d})=E_{\theta \mid x}\left(\theta^{2}\right)-\left\{E_{\theta \mid x}(\theta)\right\}^{2}$, respectively. Similarly, the Bayes estimators and posterior risks with PLF and DLF are given by:

$$
\hat{\mathrm{d}}=\left\{\mathrm{E}_{\theta \mid \mathrm{x}}\left(\theta^{2}\right)\right\}^{\frac{1}{2}}, \rho(\hat{\mathrm{d}})=2\left\{\mathrm{E}_{\theta \mid \mathrm{x}}\left(\theta^{2}\right)\right\}^{\frac{1}{2}}-2 \mathrm{E}_{\theta \mid \mathrm{x}}(\theta)
$$

And

$$
\hat{\mathrm{d}}=\frac{\mathrm{E}_{\theta \mid \mathrm{x}}\left(\theta^{2}\right)}{\mathrm{E}_{\theta \mid \mathrm{x}}(\theta)}, \rho(\hat{\mathrm{d}})=1-\frac{\left\{\mathrm{E}_{\theta \mid \mathrm{x}}(\theta)\right\}^{2}}{\mathrm{E}_{\theta \mid \mathrm{x}}\left(\theta^{2}\right)}, \text { respectively. }
$$

\subsection{The bayes estimators and posterior risks using the UP, the JP, the GP and the EP under SELF}

The Bayes estimators and posterior risks using the UP, the JP and IP for parameters $\theta_{1}, \theta_{2}, \theta_{3}, \mathrm{p}_{1}$ and $\mathrm{p}_{2}$ under SELF are obtained with their respective marginal posterior distributions are given below:

$$
\begin{aligned}
& \hat{\theta}_{1 \omega}=\Omega_{\omega}^{-1} \sum_{i=0}^{n-r} \sum_{j=0}^{i} \sum_{1=0}^{j}(-1)^{i}\left(\begin{array}{l}
n-r \\
i
\end{array}\right)\left(\begin{array}{l}
i \\
j
\end{array}\right)\left(\begin{array}{l}
j \\
1
\end{array}\right) \\
& \frac{\Gamma\left(\mathrm{A}_{1 \omega}+1\right)}{\mathrm{B}_{1 \omega}^{\mathrm{A}_{1 \omega}+1}} \frac{\Gamma\left(\mathrm{A}_{2 \omega}\right)}{\mathrm{B}_{2 \omega}^{\mathrm{A}_{2 \omega}}} \frac{\Gamma\left(\mathrm{A}_{3 \omega}\right)}{\mathrm{B}_{3 \omega}^{\mathrm{A}_{3 \omega}}} \\
& \mathrm{B}\left(\mathrm{A}_{0 \omega}, \mathrm{C}_{0 \omega}\right) \mathrm{B}\left(\mathrm{B}_{0 \omega}, \mathrm{A}_{0 \omega}+\mathrm{C}_{0 \omega}\right) \\
& \hat{\theta}_{2 \omega}=\Omega_{\omega}^{-1} \sum_{i=0}^{n-r} \sum_{j=0}^{i} \sum_{1=0}^{j}(-1)^{i}\left(\begin{array}{l}
n-r \\
i
\end{array}\right)\left(\begin{array}{l}
i \\
j
\end{array}\right)\left(\begin{array}{l}
j \\
1
\end{array}\right) \\
& \frac{\Gamma\left(\mathrm{A}_{1 \omega}\right)}{\mathrm{B}_{1 \omega}^{\mathrm{A}_{1 \omega}}} \frac{\Gamma\left(\mathrm{A}_{2 \omega}+1\right)}{\mathrm{B}_{2 \omega}^{\mathrm{A}_{2 \omega}+1}} \frac{\Gamma\left(\mathrm{A}_{3 \omega}\right)}{\mathrm{B}_{3 \omega}^{\mathrm{A}_{3 \omega}}} \mathrm{B}\left(\mathrm{A}_{0 \omega}, \mathrm{C}_{0 \omega}\right) \mathrm{B}\left(\mathrm{B}_{0 \omega}, \mathrm{A}_{0 \omega}+\mathrm{C}_{0 \omega}\right) \\
& \hat{\theta}_{3 \omega}=\Omega_{\omega}^{-1} \sum_{i=0}^{n-r} \sum_{j=01=0}^{i} \sum_{j}^{j}(-1)^{i}\left(\begin{array}{l}
n-r \\
i
\end{array}\right)\left(\begin{array}{l}
i \\
j
\end{array}\right)\left(\begin{array}{l}
j \\
1
\end{array}\right) \\
& \frac{\Gamma\left(\mathrm{A}_{1 \omega}\right)}{\mathrm{B}_{1 \omega}^{\mathrm{A}_{1 \omega}}} \frac{\Gamma\left(\mathrm{A}_{2 \omega}\right)}{\mathrm{B}_{2 \omega}^{\mathrm{A}_{2 \omega}}} \frac{\Gamma\left(\mathrm{A}_{3 \omega}+1\right)}{\mathrm{B}_{3 \omega}^{\mathrm{A}_{3 \omega}+1}} \mathrm{~B}\left(\mathrm{~A}_{0 \omega}, \mathrm{C}_{0 \omega}\right) \mathrm{B}\left(\mathrm{B}_{0 \omega}, \mathrm{A}_{0 \omega}+\mathrm{C}_{0 \omega}\right)
\end{aligned}
$$

$\hat{\mathrm{p}}_{1 \omega}=\Omega_{\omega}^{-1} \sum_{\mathrm{i}=0}^{\mathrm{n}-\mathrm{r}} \sum_{\mathrm{j}=0}^{\mathrm{i}} \sum_{1=0}^{\mathrm{j}}(-1)^{\mathrm{i}}\left(\begin{array}{l}\mathrm{n}-\mathrm{r} \\ \mathrm{i}\end{array}\right)\left(\begin{array}{l}\mathrm{i} \\ \mathrm{j}\end{array}\right)\left(\begin{array}{l}\mathrm{j} \\ 1\end{array}\right)$
$\frac{\Gamma\left(\mathrm{A}_{1 \omega}\right)}{\mathrm{B}_{1 \omega}^{\mathrm{A}_{1 \omega}}} \frac{\Gamma\left(\mathrm{A}_{2 \omega}\right)}{\mathrm{B}_{2 \omega}^{\mathrm{A}_{2 \omega}}} \frac{\Gamma\left(\mathrm{A}_{3 \omega}\right)}{\mathrm{B}_{3 \omega}^{\mathrm{A}_{3 \omega}}} \mathrm{B}\left(\mathrm{B}_{0 \omega}, \mathrm{C}_{0 \omega}\right) \mathrm{B}\left(\mathrm{A}_{0 \omega}+1, \mathrm{~B}_{0 \omega}+\mathrm{C}_{0 \omega}\right)$

$\hat{p}_{2 \omega}=\Omega_{\omega}^{-1} \sum_{i=0}^{n-r} \sum_{j=01}^{i} \sum_{1=0}^{j}(-1)^{i}\left(\begin{array}{l}n-r \\ i\end{array}\right)\left(\begin{array}{l}i \\ j\end{array}\right)\left(\begin{array}{l}j \\ 1\end{array}\right)$

$\frac{\Gamma\left(\mathrm{A}_{1 \omega}\right)}{\mathrm{B}_{1 \omega}^{\mathrm{A}_{1 \omega}}} \frac{\Gamma\left(\mathrm{A}_{2 \omega}\right)}{\mathrm{B}_{2 \omega}^{\mathrm{A}_{2 \omega}}} \frac{\Gamma\left(\mathrm{A}_{3 \omega}\right)}{\mathrm{B}_{3 \omega}^{\mathrm{A}_{3 \omega}}} \mathrm{B}\left(\mathrm{A}_{0 \omega}, \mathrm{C}_{0 \omega}\right) \mathrm{B}\left(\mathrm{B}_{0 \omega}+1, \mathrm{~A}_{0 \omega}+\mathrm{C}_{0 \omega}\right)$

$\rho\left(\hat{\theta}_{1 \omega}\right)=\Omega_{\omega}^{-1} \sum_{\mathrm{i}=0}^{\mathrm{n}-\mathrm{r}} \sum_{\mathrm{j}=0}^{\mathrm{i}} \sum_{1=0}^{\mathrm{j}}(-1)^{\mathrm{i}}\left(\begin{array}{l}\mathrm{n}-\mathrm{r} \\ \mathrm{i}\end{array}\right)\left(\begin{array}{l}\mathrm{i} \\ \mathrm{j}\end{array}\right)\left(\begin{array}{l}\mathrm{j} \\ 1\end{array}\right)$

$\frac{\Gamma\left(\mathrm{A}_{1 \omega}+2\right)}{\mathrm{B}_{1 \omega}^{\mathrm{A}_{1 \omega}+2}} \frac{\Gamma\left(\mathrm{A}_{2 \omega}\right)}{\mathrm{B}_{2 \omega}^{\mathrm{A}_{2 \omega}}} \frac{\Gamma\left(\mathrm{A}_{3 \omega}\right)}{\mathrm{B}_{3 \omega}^{\mathrm{A}_{3 \omega}}} \mathrm{B}\left(\mathrm{A}_{0 \omega}, \mathrm{C}_{0 \omega}\right)$

$\mathrm{B}\left(\mathrm{B}_{0 \omega}, \mathrm{A}_{0 \omega}+\mathrm{C}_{0 \omega}\right)-\left(\hat{\theta}_{1 \omega}\right)^{2}$

$\rho\left(\hat{\theta}_{2 \omega}\right)=\Omega_{\omega}^{-1} \sum_{i=0}^{n-r} \sum_{j=0 l=0}^{i} \sum_{j}^{j}(-1)^{i}\left(\begin{array}{l}n-r \\ i\end{array}\right)\left(\begin{array}{l}i \\ j\end{array}\right)\left(\begin{array}{l}j \\ 1\end{array}\right)$

$\frac{\Gamma\left(\mathrm{A}_{1 \omega}\right)}{\mathrm{B}_{1 \omega}^{\mathrm{A}_{1 \omega}}} \frac{\Gamma\left(\mathrm{A}_{2 \omega}+2\right)}{\mathrm{B}_{2 \omega}^{\mathrm{A}_{2 \omega}}+2} \frac{\Gamma\left(\mathrm{A}_{3 \omega}\right)}{\mathrm{B}_{3 \omega}^{\mathrm{A}_{3 \omega}}} \mathrm{B}\left(\mathrm{A}_{0 \omega}, \mathrm{C}_{0 \omega}\right)$

$\mathrm{B}\left(\mathrm{B}_{0 \omega}, \mathrm{A}_{0 \omega}+\mathrm{C}_{0 \omega}\right)-\left(\hat{\theta}_{2 \omega}\right)^{2}$

$\rho\left(\hat{\theta}_{3 \omega}\right)=\Omega_{\omega}^{-1} \sum_{i=0}^{n-r} \sum_{j=0}^{i} \sum_{l=0}^{j}(-1)^{i}\left(\begin{array}{l}n-r \\ i\end{array}\right)\left(\begin{array}{l}i \\ j\end{array}\right)\left(\begin{array}{l}j \\ 1\end{array}\right)$

$\frac{\Gamma\left(\mathrm{A}_{1 \omega}\right)}{\mathrm{B}_{1 \omega}^{\mathrm{A}_{1 \omega}}} \frac{\Gamma\left(\mathrm{A}_{2 \omega}\right)}{\mathrm{B}_{2 \omega}^{\mathrm{A}_{2 \omega}}} \frac{\Gamma\left(\mathrm{A}_{3 \omega}+2\right)}{\mathrm{B}_{3 \omega}^{\mathrm{A}_{3 \omega}+2}}$

$\mathrm{B}\left(\mathrm{A}_{0 \omega}, \mathrm{C}_{0 \omega}\right) \mathrm{B}\left(\mathrm{B}_{0 \omega}, \mathrm{A}_{0 \omega}+\mathrm{C}_{0 \omega}\right)-\left(\hat{\theta}_{3 \omega}\right)^{2}$ 


$$
\begin{aligned}
& \rho\left(\hat{\mathrm{p}}_{1 \omega}\right)=\Omega_{\omega}^{-1} \sum_{\mathrm{i}=0}^{\mathrm{n}-\mathrm{r}} \sum_{\mathrm{j}=01=0}^{\mathrm{i}} \sum_{1=0}^{\mathrm{j}}(-1)^{\mathrm{i}}\left(\begin{array}{l}
\mathrm{n}-\mathrm{r} \\
\mathrm{i}
\end{array}\right)\left(\begin{array}{l}
\mathrm{i} \\
\mathrm{j}
\end{array}\right)\left(\begin{array}{l}
\mathrm{j} \\
1
\end{array}\right) \\
& \frac{\Gamma\left(\mathrm{A}_{1 \omega}\right)}{\mathrm{B}_{1 \omega}^{\mathrm{A}_{1}}} \frac{\Gamma\left(\mathrm{A}_{2 \omega}\right)}{\mathrm{B}_{2 \omega}} \frac{\Gamma\left(\mathrm{A}_{3 \omega}\right)}{\mathrm{A}_{3 \omega}}
\end{aligned}
$$$$
\mathrm{B}\left(\mathrm{B}_{0 \omega}, \mathrm{C}_{0 \omega}\right) \mathrm{B}\left(\mathrm{A}_{0 \omega}+2, \mathrm{~B}_{0 \omega}+\mathrm{C}_{0 \omega}\right)-\left(\hat{\mathrm{p}}_{1 \omega}\right)^{2}
$$$$
\rho\left(\hat{p}_{2 \omega}\right)=\Omega_{\omega}^{-1} \sum_{i=0}^{n-r} \sum_{j=01=0}^{i} \sum_{1}^{j}(-1)^{i}\left(\begin{array}{l}
n-r \\
i
\end{array}\right)\left(\begin{array}{l}
i \\
j
\end{array}\right)\left(\begin{array}{l}
j \\
1
\end{array}\right)
$$$$
\frac{\Gamma\left(\mathrm{A}_{1 \omega}\right)}{\mathrm{B}_{1 \omega}^{\mathrm{A}_{1 \omega}}} \frac{\Gamma\left(\mathrm{A}_{2 \omega}\right)}{\mathrm{B}_{2 \omega}^{\mathrm{A}_{2 \omega}}} \frac{\Gamma\left(\mathrm{A}_{3 \omega}\right)}{\mathrm{B}_{3 \omega}^{\mathrm{A}_{3 \omega}}}
$$

$\mathrm{B}\left(\mathrm{A}_{0 \omega}, \mathrm{C}_{0 \omega}\right) \mathrm{B}\left(\mathrm{B}_{0 \omega}+2, \mathrm{~A}_{0 \omega}+\mathrm{C}_{0 \omega}\right)-\left(\hat{\mathrm{p}}_{2 \omega}\right)^{2}$

Where $\omega=1$ for the UP, $\omega=2$ for the JP, $\omega=3$ for the Gamma prior and $\omega=4$ for the Exponential prior.

\subsection{The bayes estimators and posterior risks using the UP, the JP, the GP and the EP under PLF}

Norstrom discussed an asymmetric PLF and also introduced a special case of general class of PLFs, which is defined as $L(\theta, d)=\frac{(\theta-d)^{2}}{d}$. The Bayes estimator and posterior risk are: $\hat{\mathrm{d}}=\left\{\mathrm{E}_{\theta \mid \mathrm{x}}\left(\theta^{2}\right)\right\}^{\frac{1}{2}}, \rho(\hat{\mathrm{d}})=2\left\{\mathrm{E}_{\theta \mid \mathrm{x}}\left(\theta^{2}\right)\right\}^{\frac{1}{2}}-2 \mathrm{E}_{\theta \mid \mathrm{x}}(\theta)$, respectively. The respective marginal posterior distribution yields the Bayes estimators and posterior risk using the UP, the JP and the IP for parameters $\theta_{1}, \theta_{2}, \theta_{3}, \mathrm{p}_{1}$ and $\mathrm{p}_{2}$ under PLF as:

$\hat{\theta}_{1 \omega}=$

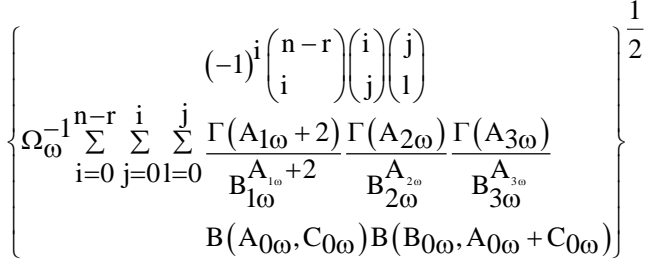

$\hat{\theta}_{2 \omega}=$

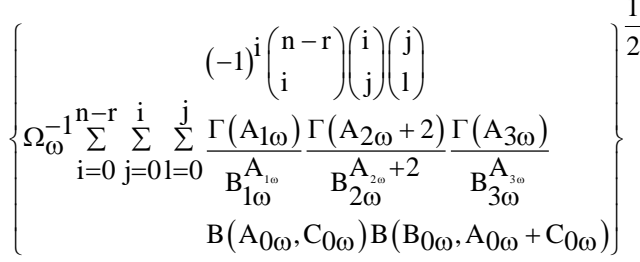

$$
\begin{aligned}
& \hat{\theta}_{3 \omega}= \\
& \left\{\begin{array}{c}
(-1)^{\mathrm{i}(}\left(\begin{array}{l}
\mathrm{n}-\mathrm{r} \\
\mathrm{i}
\end{array}\right)\left(\begin{array}{l}
\mathrm{i} \\
\mathrm{j}
\end{array}\right)\left(\begin{array}{l}
\mathrm{j} \\
1
\end{array}\right) \\
\Omega_{\omega}^{-1} \sum_{\mathrm{i}=0}^{\mathrm{n}-\mathrm{r}} \sum_{\mathrm{j}=01=0}^{\mathrm{i}} \sum_{1=0}^{\mathrm{j}} \frac{\Gamma\left(\mathrm{A}_{1 \omega}\right)}{\mathrm{B}_{1 \omega}^{\mathrm{A}_{1 \omega}}} \frac{\Gamma\left(\mathrm{A}_{2 \omega}\right)}{\mathrm{B}_{2 \omega}^{\mathrm{A}_{2 \omega}}} \frac{\Gamma\left(\mathrm{A}_{3 \omega}+2\right)}{\mathrm{B}_{3 \omega}^{\mathrm{A}_{3 \omega}+2}} \\
\mathrm{~B}\left(\mathrm{~A}_{0 \omega}, \mathrm{C}_{0 \omega}\right) \mathrm{B}\left(\mathrm{B}_{0 \omega}, \mathrm{A}_{0 \omega}+\mathrm{C}_{0 \omega}\right)
\end{array}\right\}
\end{aligned}
$$

$$
\hat{\mathrm{p}} 1 \omega=\left\{\begin{array}{c}
(-1)^{\mathrm{i}}\left(\begin{array}{l}
\mathrm{n}-\mathrm{r} \\
\mathrm{i}
\end{array}\right)\left(\begin{array}{l}
\mathrm{i} \\
\mathrm{j}
\end{array}\right)\left(\begin{array}{l}
\mathrm{j} \\
1
\end{array}\right) \\
\Omega_{\mathrm{i}=0}^{-1} \sum_{\mathrm{j}=01=0}^{\mathrm{n}-\mathrm{r}} \frac{\sum_{1}^{\mathrm{i}} \sum_{1 \omega}^{\mathrm{j}} \frac{\Gamma\left(\mathrm{A}_{1 \omega}\right)}{\mathrm{B}_{1 \omega}^{\mathrm{A}_{1 \omega}}} \frac{\Gamma\left(\mathrm{A}_{2 \omega}\right)}{\mathrm{B}_{2 \omega}} \frac{\Gamma\left(\mathrm{A}_{3 \omega}\right)}{\mathrm{B}_{3 \omega}^{\mathrm{A}_{3 \omega}}}}{\mathrm{B}\left(\mathrm{B}_{0 \omega}, \mathrm{C}_{0 \omega}\right) \mathrm{B}\left(\mathrm{A}_{0 \omega}+2, \mathrm{~B}_{0 \omega}+\mathrm{C}_{0 \omega}\right)}
\end{array}\right\}
$$

$$
\hat{p}_{2 \omega}=\left\{\begin{array}{c}
(-1)^{\mathrm{i}}\left(\begin{array}{l}
\mathrm{n}-\mathrm{r} \\
\mathrm{i}
\end{array}\right)\left(\begin{array}{l}
\mathrm{i} \\
\mathrm{j}
\end{array}\right)\left(\begin{array}{l}
\mathrm{j} \\
1
\end{array}\right) \\
\sum_{\mathrm{i}=0}^{\mathrm{n}-\mathrm{r}} \sum_{\mathrm{j}=01=0}^{\mathrm{i}} \sum_{1=0}^{\mathrm{j}} \frac{\Gamma\left(\mathrm{A}_{1 \omega}\right)}{\mathrm{B}_{1 \omega}^{\mathrm{A}_{1 \omega}}} \frac{\Gamma\left(\mathrm{A}_{2 \omega}\right)}{\mathrm{B}_{2 \omega}^{\mathrm{A}_{2 \omega}}} \frac{\Gamma\left(\mathrm{A}_{3 \omega}\right)}{\mathrm{B}_{3 \omega}^{\mathrm{A}_{3 \omega}}} \\
\mathrm{B}\left(\mathrm{A}_{0 \omega}, \mathrm{C}_{0 \omega}\right) \mathrm{B}\left(\mathrm{B}_{0 \omega}+2, \mathrm{~A}_{0 \omega}+\mathrm{C}_{0 \omega}\right)
\end{array}\right\}
$$

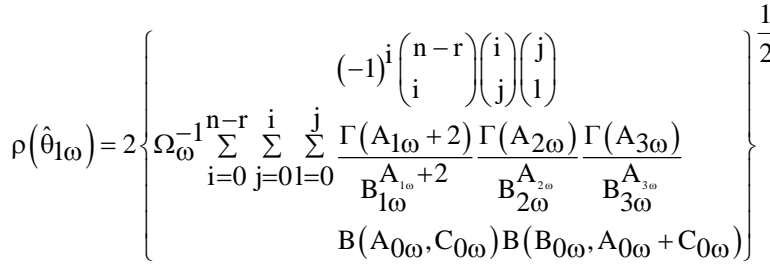

$$
\begin{aligned}
& -2\left\{\begin{array}{c}
\Omega_{\omega}^{-1} \sum_{i=0}^{n-r} \sum_{j=0}^{i} \sum_{1=0}^{j} \frac{(-1)^{i}\left(\begin{array}{l}
n-r \\
i
\end{array}\right)\left(\begin{array}{l}
i \\
j
\end{array}\right)\left(\begin{array}{l}
j \\
1
\end{array}\right)}{B_{1 \omega}^{A_{1 \omega}+1}} \frac{\Gamma\left(A_{2 \omega}\right)}{B_{2 \omega}^{A_{2 \omega}}} \frac{\Gamma\left(A_{3 \omega}\right)}{B_{3 \omega}^{A_{3 \omega}}} \\
B\left(A_{0 \omega}, C_{0 \omega}\right) B\left(B_{0 \omega}, A_{0 \omega}+C_{0 \omega}\right)
\end{array}\right\}
\end{aligned}
$$

$$
\begin{aligned}
& \rho\left(\hat{\theta}_{2 \omega}\right)=2\left\{\begin{array}{c}
(-1)^{\mathrm{i}}\left(\begin{array}{l}
\mathrm{n}-\mathrm{r} \\
\mathrm{i}
\end{array}\right)\left(\begin{array}{l}
\mathrm{i} \\
\mathrm{j}
\end{array}\right)\left(\begin{array}{l}
\mathrm{j} \\
1
\end{array}\right) \\
\Omega_{\omega}^{-1} \sum_{\mathrm{i}=0}^{\mathrm{n}-\mathrm{r}} \sum_{\mathrm{j}=01=0}^{\mathrm{i}} \sum_{1=0}^{\mathrm{j}} \frac{\Gamma\left(\mathrm{A}_{1 \omega}\right)}{\mathrm{B}_{1 \omega}^{\mathrm{A}_{1 \omega}}} \frac{\Gamma\left(\mathrm{A}_{2 \omega}+2\right)}{\mathrm{B}_{2 \omega}^{\mathrm{A}_{2 \omega}+2}} \frac{\Gamma\left(\mathrm{A}_{3 \omega}\right)}{\mathrm{B}_{3 \omega}^{\mathrm{A}_{3 \omega}}} \\
\mathrm{B}\left(\mathrm{A}_{0 \omega}, \mathrm{C}_{0 \omega}\right) \mathrm{B}\left(\mathrm{B}_{0 \omega}, \mathrm{A}_{0 \omega}+\mathrm{C}_{0 \omega}\right)
\end{array}\right\}
\end{aligned}
$$

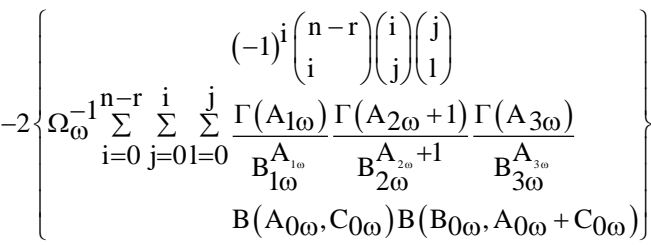

$$
\begin{aligned}
& (-1)^{i}\left(\begin{array}{l}
n-r \\
i
\end{array}\right)\left(\begin{array}{l}
i \\
j
\end{array}\right)\left(\begin{array}{l}
j \\
1
\end{array}\right) \\
& \rho\left(\hat{\theta}_{3 \omega}\right)=2\left\{\Omega_{\omega}^{-1} \sum_{\mathrm{i}=0}^{\mathrm{n}-\mathrm{r}} \sum_{\mathrm{j}=01=0}^{\mathrm{i}} \sum_{1=0}^{\mathrm{j}} \frac{\Gamma\left(\mathrm{A}_{1 \omega}\right)}{\mathrm{B}_{1 \omega}^{\mathrm{A}_{1 \omega}}} \frac{\Gamma\left(\mathrm{A}_{2 \omega}\right)}{\mathrm{B}_{2 \omega}^{\mathrm{A}_{2 \omega}}} \frac{\Gamma\left(\mathrm{A}_{3 \omega}+2\right)}{\mathrm{B}_{3 \omega}^{\mathrm{A}_{3 \omega}+2}}\right. \\
& \mathrm{B}\left(\mathrm{A}_{0 \omega}, \mathrm{C}_{0 \omega}\right) \mathrm{B}\left(\mathrm{B}_{0 \omega}, \mathrm{A}_{0 \omega}+\mathrm{C}_{0 \omega}\right) \\
& (-1)^{\mathrm{i}}\left(\begin{array}{l}
\mathrm{n}-\mathrm{r} \\
\mathrm{i}
\end{array}\right)\left(\begin{array}{l}
\mathrm{i} \\
\mathrm{j}
\end{array}\right)\left(\begin{array}{l}
\mathrm{j} \\
1
\end{array}\right) \\
& \left.\begin{array}{rl}
-2\left\{\Omega_{\omega}^{-1} \sum_{\mathrm{i}=0}^{\mathrm{n}-\mathrm{r}} \sum_{\mathrm{j}=01}^{\mathrm{i}} \sum_{1=0}^{\mathrm{j}} \frac{\Gamma\left(\mathrm{A}_{1 \omega}\right)}{\mathrm{B}_{1 \omega}^{\mathrm{A}_{1 \omega}}} \frac{\Gamma\left(\mathrm{A}_{2 \omega}\right)}{\mathrm{B}_{2 \omega}^{\mathrm{A}_{2 \omega}}} \frac{\Gamma\left(\mathrm{A}_{3 \omega}+1\right)}{\mathrm{B}_{3 \omega}^{\mathrm{A}_{3 \omega}+1}}\right. \\
\mathrm{B}\left(\mathrm{A}_{0 \omega}, \mathrm{C}_{0 \omega}\right) \mathrm{B}\left(\mathrm{B}_{0 \omega}, \mathrm{A}_{0 \omega}+\mathrm{C}_{0 \omega}\right)
\end{array}\right\}
\end{aligned}
$$


$\rho\left(\hat{\mathrm{p}}_{1 \omega}\right)=$

$$
\begin{aligned}
& 2\left\{\begin{array}{c}
\Omega_{\omega}^{-1} \sum_{\mathrm{i}=0}^{\mathrm{n}-\mathrm{r}} \sum_{\mathrm{j}=01=0}^{\mathrm{i}} \sum_{1=0}^{\mathrm{j}} \frac{(-1)^{\mathrm{i}}\left(\begin{array}{l}
\mathrm{n}-\mathrm{r} \\
\mathrm{i}
\end{array}\right)\left(\begin{array}{l}
\mathrm{i} \\
\mathrm{j}
\end{array}\right)\left(\begin{array}{l}
\mathrm{j} \\
1
\end{array}\right)}{\left.\mathrm{B}_{1 \omega}\right)} \frac{\Gamma\left(\mathrm{A}_{2 \omega}\right)}{\mathrm{B}_{2 \omega}} \frac{\Gamma\left(\mathrm{A}_{3 \omega}\right)}{\mathrm{B}_{3 \omega}} \\
\mathrm{B}\left(\mathrm{B}_{0 \omega}, \mathrm{C}_{0 \omega}\right) \mathrm{B}\left(\mathrm{A}_{0 \omega}+2, \mathrm{~B}_{0 \omega}+\mathrm{C}_{0 \omega}\right)
\end{array}\right\} \\
& -2\left\{\begin{array}{c}
\Omega_{\omega}^{-1} \sum_{i=0}^{n-r} \sum_{j=01}^{i} \sum_{=0}^{j} \frac{\Gamma\left(A_{1 \omega}\right)}{B_{1 \omega}^{A_{1 \omega}}} \frac{\Gamma\left(\begin{array}{l}
n-r \\
i
\end{array}\right)\left(\begin{array}{l}
i \\
j
\end{array}\right)\left(\begin{array}{l}
j \\
1
\end{array}\right)}{B_{2 \omega}^{A_{2 \omega}}} \frac{\Gamma\left(A_{3 \omega}\right)}{B_{3 \omega}^{A_{3 \omega}}} \\
B\left(B_{0 \omega}, C_{0 \omega}\right) B\left(A_{0 \omega}+1, B_{0 \omega}+C_{0 \omega}\right)
\end{array}\right\}
\end{aligned}
$$

$\rho\left(\hat{\mathrm{p}}_{2 \omega}\right)=$

$$
\begin{aligned}
& 2\left\{\begin{array}{c}
\Omega_{\omega}^{-1} \sum_{i=0}^{n-r} \sum_{j=01=0}^{i} \frac{(-1)^{i}\left(\begin{array}{l}
n-r \\
i
\end{array}\right)\left(\begin{array}{l}
i \\
j
\end{array}\right)\left(\begin{array}{l}
j \\
1
\end{array}\right)}{B_{1 \omega}^{A_{1 \omega}}} \frac{\Gamma\left(A_{2 \omega}\right)}{B_{2 \omega}^{A_{2 \omega}}} \frac{\Gamma\left(A_{3 \omega}\right)}{B_{3 \omega}^{A_{3 \omega}}} \\
B\left(A_{0 \omega}, C_{0 \omega}\right) B\left(B_{0 \omega}+2, A_{0 \omega}+C_{0 \omega}\right)
\end{array}\right\} \\
& -2\left\{\begin{array}{c}
\Omega_{\omega}^{-1} \sum_{i=0}^{\mathrm{n}-\mathrm{r}} \sum_{\mathrm{j}=01=0}^{\mathrm{i}} \sum_{1=0}^{\mathrm{j}} \frac{\Gamma\left(\mathrm{A}_{1 \omega}\right)}{\mathrm{B}_{1 \omega}^{\mathrm{A}_{1 \omega}}} \frac{\Gamma\left(\begin{array}{l}
\mathrm{n}-\mathrm{r} \\
\mathrm{i}
\end{array}\right)\left(\begin{array}{l}
\mathrm{i} \\
\mathrm{j}
\end{array}\right)\left(\begin{array}{l}
\mathrm{j} \\
\mathrm{A}
\end{array}\right)}{\left.\mathrm{B}_{2 \omega}\right)} \frac{\Gamma\left(\mathrm{A}_{3 \omega}\right)}{\mathrm{B}_{3 \omega}^{\mathrm{A}_{3 \omega}}} \\
\mathrm{B}\left(\mathrm{A}_{0 \omega}, \mathrm{C}_{0 \omega}\right) \mathrm{B}\left(\mathrm{B}_{0 \omega}+1, \mathrm{~A}_{0 \omega}+\mathrm{C}_{0 \omega}\right)
\end{array}\right\}
\end{aligned}
$$

Where $\omega=1$ for the UP, $\omega=2$ for the JP, $\omega=3$ for the Gamma prior and $\omega=4$ for the Exponential prior.

\subsection{The bayes estimators and posterior risks using the UP, the JP, the GP and the EP under DLF}

DeGroot (2005) introduced the asymmetric loss function, $\mathrm{L}(\theta, \mathrm{d})=\left(\frac{\theta-\mathrm{d}}{\mathrm{d}}\right)^{2}$ known as DLF. The Bayes estimator and its posterior risk under DLF are: $\hat{\mathrm{d}}=\frac{\mathrm{E}_{\theta \mid \mathrm{x}}\left(\theta^{2}\right)}{\mathrm{E}_{\theta \mid \mathrm{x}}(\theta)} \quad$ and $\rho(\hat{d})=1-\frac{\left\{E_{\theta \mid X}(\theta)\right\}^{2}}{E_{\theta \mid X}\left(\theta^{2}\right)}$, respectively. The Bayes estimators and posterior risks using the UP,the JP and the IP for parameters $\theta_{1}, \theta_{2}, \theta_{3}, \mathrm{p}_{1}$ and $\mathrm{p}_{2}$ under DLF are:

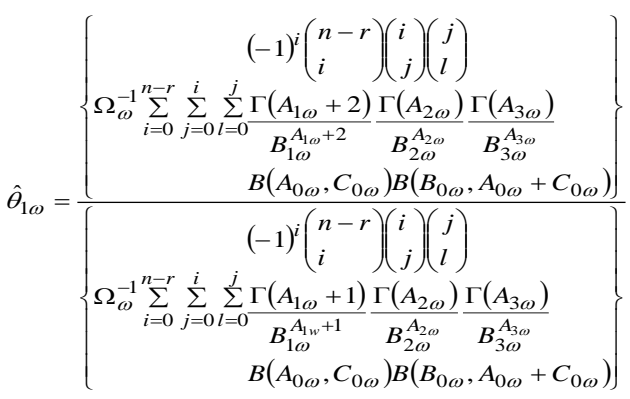

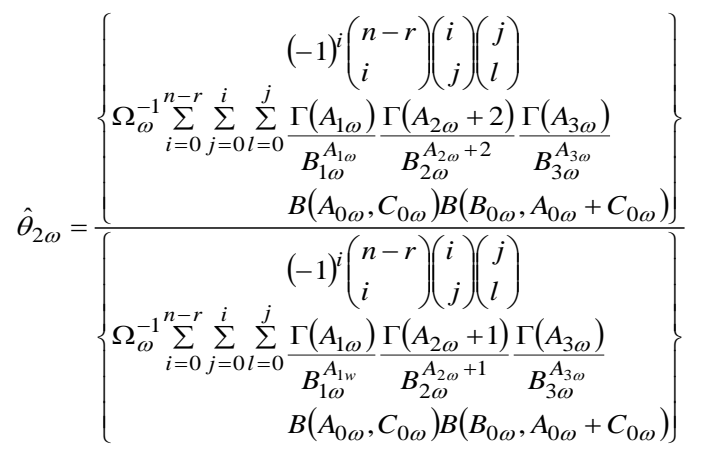

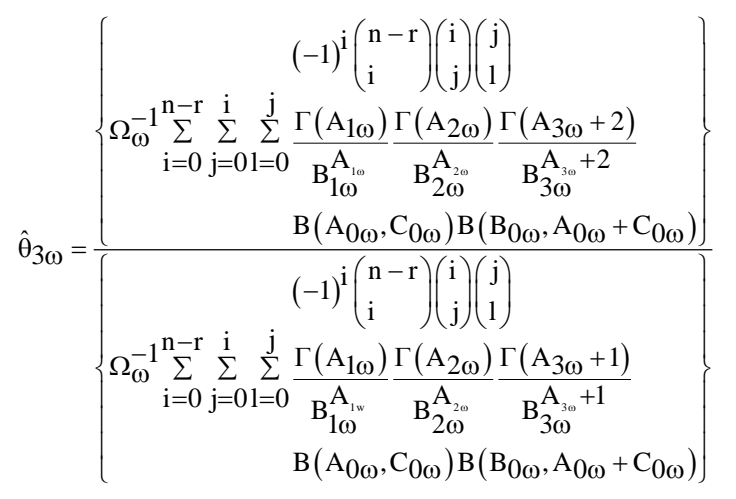

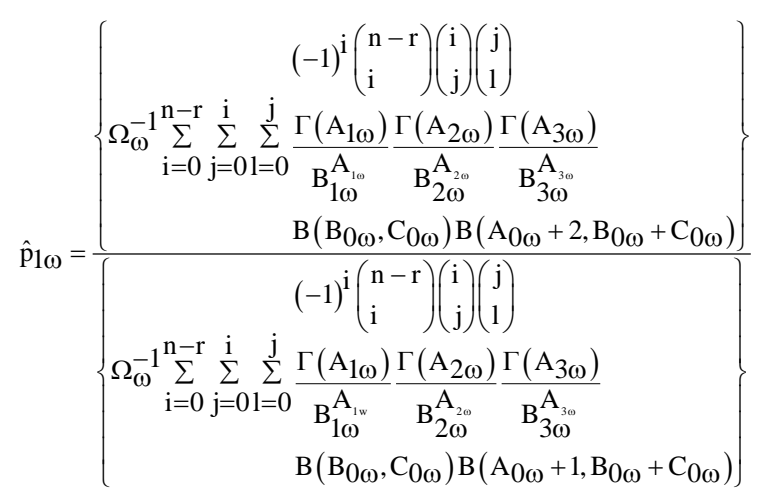

$$
\begin{aligned}
& (-1)^{\mathrm{i}}\left(\begin{array}{l}
\mathrm{n}-\mathrm{r} \\
\mathrm{i}
\end{array}\right)\left(\begin{array}{l}
\mathrm{i} \\
\mathrm{j}
\end{array}\right)\left(\begin{array}{l}
\mathrm{j} \\
\mathrm{l}
\end{array}\right) \\
& \left\{\Omega_{\omega}^{-1} \sum \sum \sum \sum \underline{\mathrm{n}-\mathrm{r}} \sum^{\mathrm{i}} \mathrm{A}_{1 \omega}^{\mathrm{j}}\right) \Gamma\left(\mathrm{A}_{2 \omega}\right) \Gamma\left(\mathrm{A}_{3 \omega}\right) \\
& \mathrm { i } = 0 \mathrm { j } = 0 1 = 0 \longdiv { B _ { 1 \omega } ^ { A _ { 1 \omega } } } \frac { \mathrm { B } _ { 2 \omega } ^ { A _ { 2 \omega } } } { B _ { 3 \omega } ^ { A _ { 3 \omega } } }
\end{aligned}
$$

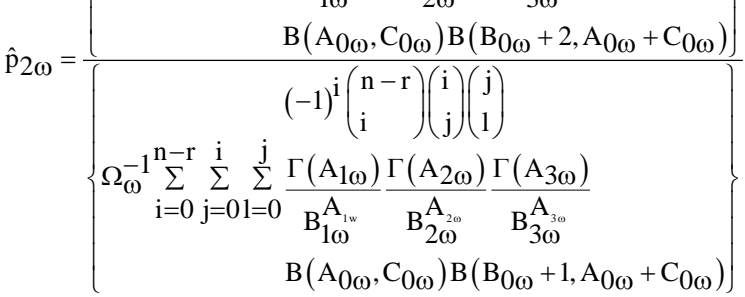


$\rho\left(\hat{\theta}_{1}\right)=$

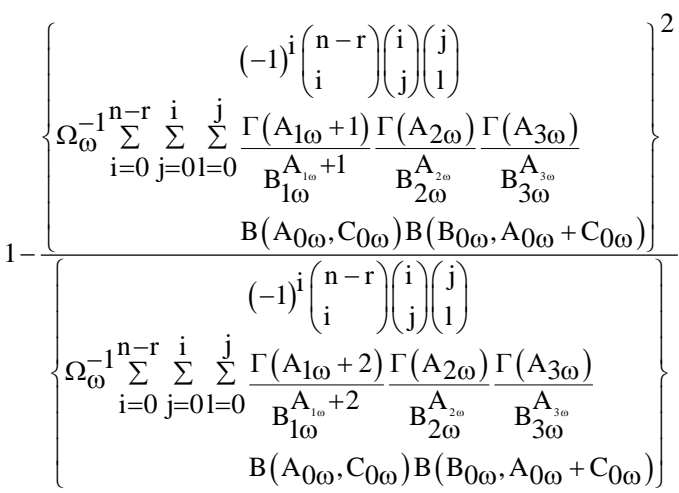

$\rho\left(\hat{\theta}_{2}\right)=$

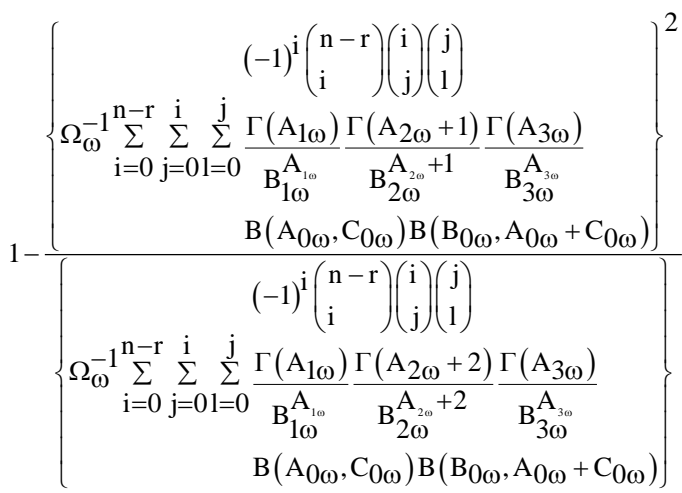

$\rho\left(\hat{\theta}_{3}\right)=$

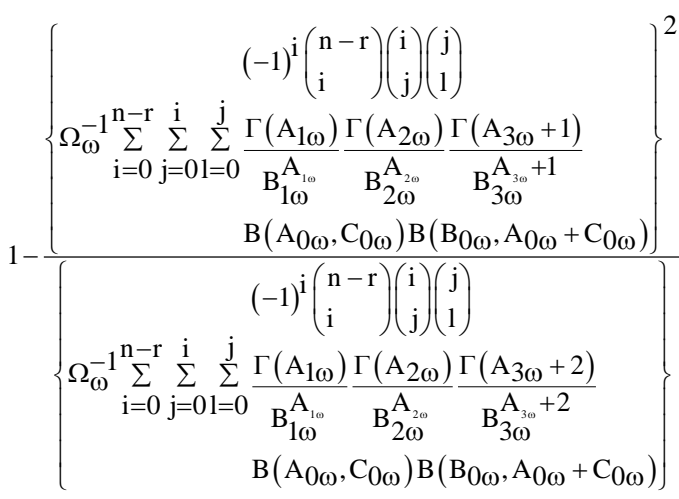

$\rho\left(\hat{\mathrm{p}}_{1}\right)=$

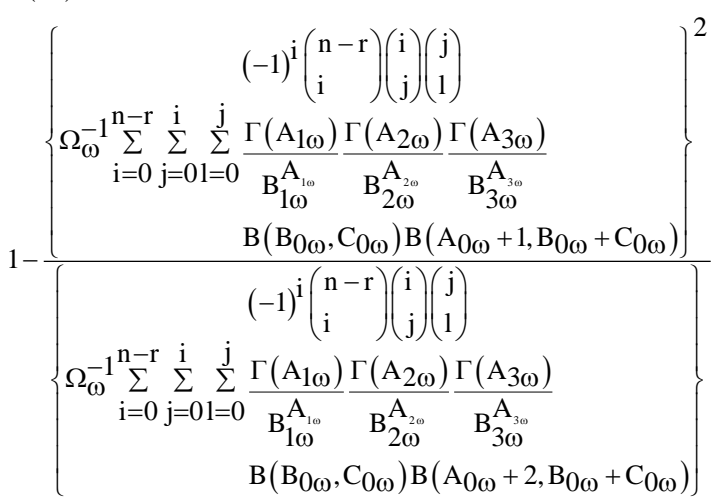

$\rho\left(\hat{\mathrm{p}}_{2}\right)=$

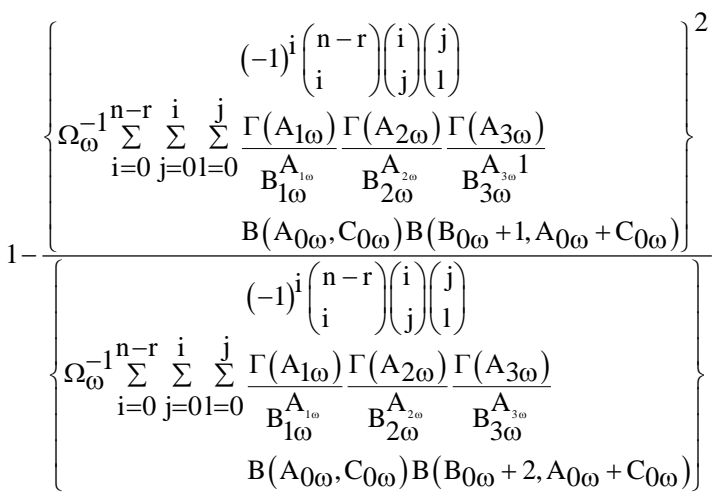

\section{Elicitation of hyper-parameters}

Elicitation is the key task for subjective Bayesian. The whole procedure for quantifying the prior information in the form of prior distribution is precisely known as the elicitation. Aslam [3] proposed different methods of elicitation based on prior predictive distribution for the elicitation of the hyper-parameters. In this study, we use the method of elicitation using prior predictive distribution based on the predictive probabilities. In this method, confidence levels of the prior predictive are obtained for the particular intervals of the random variable's'. The set of hyper parameters, for which the difference between the elicited probabilities and the expert predictive probabilities is minimum, is considered.

\subsection{Elicitation of hyper-parameters using the gamma prior}

For eliciting the hyper-parameters, prior predictive distribution (PPD) is used. The PPD for a random variable $\mathrm{X}$ is:

$$
p(x)=\int p\langle x \mid \varphi\rangle \pi_{3}(\varphi) d \varphi
$$

(48)

$$
p(x)=\frac{2}{(a+b+c) x^{3}}\left[\begin{array}{c}
\frac{a_{1} b_{1}^{a_{1}}}{\left(b_{1}+x^{-2}\right)^{a_{1}+1}} \\
+\frac{b_{2} b_{2}^{a_{2}}}{\left(b_{2}+x^{-2}\right)^{a_{2}+1}} \\
+\frac{c a_{3} b_{3}^{a_{3}}}{\left(b_{3}+x^{-2}\right)^{a_{3}+1}}
\end{array}\right]
$$

We choose the prior predictive probabilities, satisfying the laws of probability, to elicit the hyper parameters of the prior density. Using the prior predictive distribution given in (51), we consider the nine intervals $(0,1),(1,2),(2,3),(3,4),(4,5),(5,6),(6,7),(7,8)$ and $(8,9)$ with probabilities $0.18,0.29,0.20,0.11,0.07,0.04,0.03$, 0.02 and 0.01 respectively, given an expert opinion. The following nine equations are derived from the given information using (51) as:

$\frac{2}{(a+b+c) x^{3}} \int_{0}^{1}\left[\begin{array}{l}\frac{a a_{1} b_{1}^{a_{1}}}{\left(b_{1}+x^{-2}\right)^{a_{1}+1}}+\frac{b a_{2} b_{2}^{a_{2}}}{\left(b_{2}+x^{-2}\right)^{a_{2}+1}} \\ +\frac{c a_{3} b_{3}^{a_{3}}}{\left(b_{3}+x^{-2}\right)^{a_{3}+1}}\end{array}\right] d x=0.18$ 
$\frac{2}{(a+b+c) x^{3}} \int_{1}^{2}\left[\begin{array}{l}\frac{a a_{1} b_{1}^{a_{1}}}{\left(b_{1}+x^{-2}\right)^{a_{1}+1}}+\frac{b a_{2} b_{2}^{a_{2}}}{\left(b_{2}+x^{-2}\right)^{a_{2}+1}} \\ +\frac{c a_{3} b_{3}^{a_{3}}}{\left(b_{3}+x^{-2}\right)^{a_{3}+1}}\end{array}\right] d x=0.29$

$\frac{2}{(a+b+c) x^{3}} \int_{2}^{3}\left[\begin{array}{l}\frac{a a_{1} b_{1}^{a_{1}}}{\left(b_{1}+x^{-2}\right)^{a_{1}+1}}+\frac{b a_{2} b_{2}^{a_{2}}}{\left(b_{2}+x^{-2}\right)^{a_{2}+1}} \\ +\frac{c a_{3} b_{3}^{a_{3}}}{\left(b_{3}+x^{-2}\right)^{a_{3}+1}}\end{array}\right] d x=0.20$

$\frac{2}{(a+b+c) x^{3}} \int_{3}^{4}\left[\begin{array}{l}\frac{a a_{1} b_{1}^{a_{1}}}{\left(b_{1}+x^{-2}\right)^{a_{1}+1}}+\frac{b a_{2} b_{2}^{a_{2}}}{\left(b_{2}+x^{-2}\right)^{a_{2}+1}} \\ +\frac{c a_{3} b_{3}^{a_{3}}}{\left(b_{3}+x^{-2}\right)^{a_{3}+1}}\end{array}\right] d x=0.11$
$\frac{2}{(a+b+c) x^{3}} \int_{4}^{5}\left[\begin{array}{l}\frac{a a_{1} b_{1}^{a_{1}}}{\left(b_{1}+x^{-2}\right)^{a_{1}+1}}+\frac{b a_{2} b_{2}^{a_{2}}}{\left(b_{2}+x^{-2}\right)^{a_{2}+1}} \\ +\frac{c a_{3} b_{3}^{a_{3}}}{\left(b_{3}+x^{-2}\right)^{a_{3}+1}}\end{array}\right] d x=0.07$

$\frac{2}{(a+b+c) x^{3}} \int_{5}^{6}\left[\begin{array}{l}\frac{a a_{1} b_{1}^{a_{1}}}{\left(b_{1}+x^{-2}\right)^{a_{1}+1}}+\frac{b a_{2} b_{2}^{a_{2}}}{\left(b_{2}+x^{-2}\right)^{a_{2}+1}} \\ +\frac{c a_{3} b_{3}^{a_{3}}}{\left(b_{3}+x^{-2}\right)^{a_{3}+1}}\end{array}\right] d x=0.04$

$\frac{2}{(a+b+c) x^{3}} \int_{6}^{7}\left[\begin{array}{l}\frac{a a_{1} b_{1}^{a_{1}}}{\left(b_{1}+x^{-2}\right)^{a_{1}+1}}+\frac{b a_{2} b_{2}^{a_{2}}}{\left(b_{2}+x^{-2}\right)^{a_{2}+1}} \\ +\frac{c a_{3} b_{3}^{a_{3}}}{\left(b_{3}+x^{-2}\right)^{a_{3}+1}}\end{array}\right] d x=0.03$

$$
\begin{aligned}
& \frac{2}{(a+b+c) x^{3}} \int_{7}^{8}\left[\begin{array}{l}
\frac{a a_{1} b_{1}^{a_{1}}}{\left(b_{1}+x^{-2}\right)^{a_{1}+1}}+\frac{b a_{2} b_{2}^{a_{2}}}{\left(b_{2}+x^{-2}\right)^{a_{2}+1}} \\
+\frac{c a_{3} b_{3}^{a_{3}}}{\left(b_{3}+x^{-2}\right)^{a_{3}+1}}
\end{array}\right] d x=0.02 \\
& \frac{2}{(a+b+c) x^{3}} \int_{8}^{9}\left[\begin{array}{l}
\frac{a a_{1} b_{1}^{a_{1}}}{\left(b_{1}+x^{-2}\right)^{a_{1}+1}}+\frac{b a_{2} b_{2}^{a_{2}}}{\left(b_{2}+x^{-2}\right)^{a_{2}+1}} \\
+\frac{c a_{3} b_{3}^{a_{3}}}{\left(b_{3}+x^{-2}\right)^{a_{3}+1}}
\end{array}\right] d x=0.01(6
\end{aligned}
$$

For eliciting the hyper parameters $\mathrm{a}_{1}, \mathrm{a}_{2}, \mathrm{a}_{3}, \mathrm{~b}_{1}, \mathrm{~b}_{2}, \mathrm{~b}_{3}, \mathrm{a}, \mathrm{b}$ and $\mathrm{c}$, the equations are simultaneously solved through the computer program developed in SAS package using the 'PROC SYSLIN' command, the values of the hyper parameters are found to be $0.9965,2.1454,3,0.4334,0.2554,0.7450,2.4727,1.5$ and 1.0269 respectively.

\subsection{Elicitation of hyper-parameters using the Exponen- tial Prior}

The PPD using Exponential prior for a random variable $\mathrm{X}$ is given by:

$$
\begin{aligned}
& \mathrm{p}(\mathrm{x})=\int_{\varphi} \mathrm{p}\langle\mathrm{x} \mid \varphi\rangle \pi_{4}(\varphi) \mathrm{d} \varphi \\
& \mathrm{p}(\mathrm{x})=\frac{2}{\mathrm{x}^{3}(\mathrm{a}+\mathrm{b}+\mathrm{c})}\left[\begin{array}{l}
\frac{\mathrm{aw}_{1}}{\left(\mathrm{w}_{1}+\mathrm{x}^{-2}\right)^{2}}+\frac{\mathrm{bw}_{2}}{\left(\mathrm{w}_{2}+\mathrm{x}^{-2}\right)^{2}} \\
+\frac{\mathrm{cw}_{3}}{\left(\mathrm{w}_{3}+\mathrm{x}^{-2}\right)^{2}}
\end{array}\right]
\end{aligned}
$$

Using similar criteria defined as above for exponential prior, the values of the hyper-parameters $\mathrm{w}_{1}, \mathrm{w}_{2}, \mathrm{w}_{3}, \mathrm{a}, \mathrm{b}$ and $\mathrm{c}$ are 2.9607 , $1.9215,0.9942,1.1177,0.7033$ and 0.50 .

\section{Limiting expressions}

Letting $\mathrm{t} \rightarrow \infty$, all the observations that are incorporated in our analysis are uncensored and therefore $r$ tends to $n, r$ tends to the unknown $\mathrm{n}_{1}, \mathrm{r}_{2}$ tends to the unknown $\mathrm{n}_{2}$ and $\mathrm{r}_{3}$ tends to the unknown $n_{3}$. As a result, the amount of information contained in the sample is increasing, which consequently results in the reduction of the variances of the estimates. The limiting (complete sample) expressions for Bayes estimators and posterior risks using the UP, the JP and the IP under SELF, PLF and DLF are given in the Tables 1-7.

Table 1: Limiting Expressions for the Bayes Estimators as $t \rightarrow \infty$ Using the UP, the JP and the IP under SELF

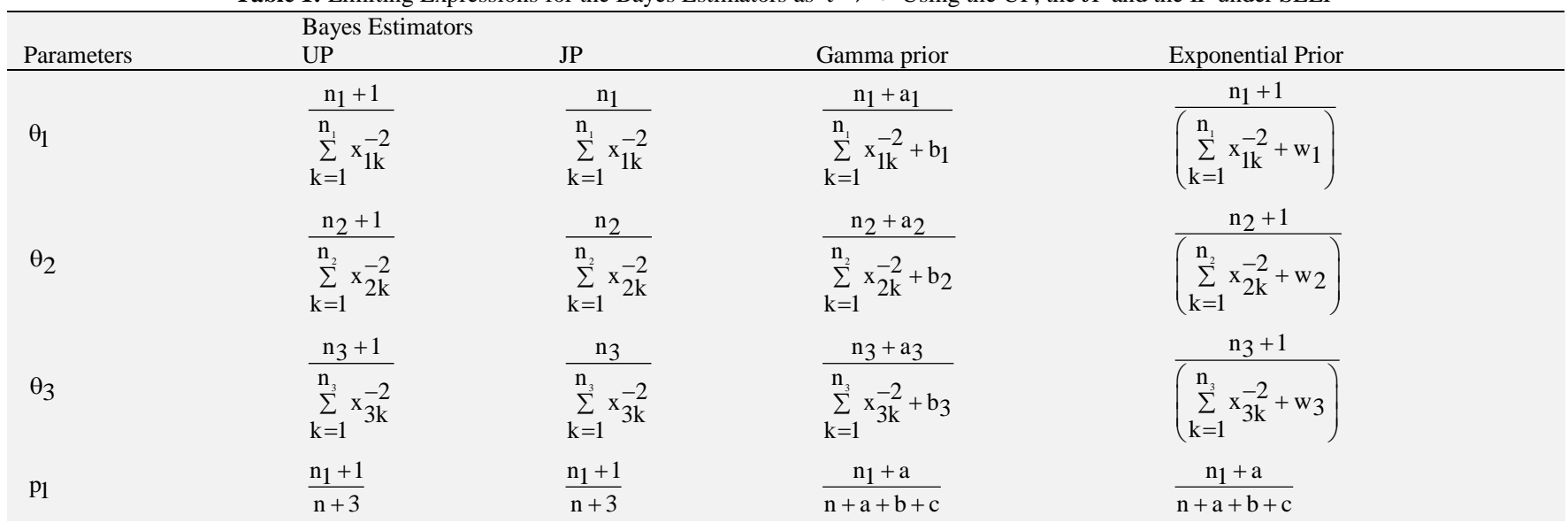




$\mathrm{p}_{2} \frac{\mathrm{n}_{2}+1}{\mathrm{n}+3} \quad \frac{\mathrm{n}_{2}+1}{\mathrm{n}+3} \quad \frac{n_{2}+b}{n+a+b+c} \quad \frac{n_{2}+b}{n+a+b+c}$

Table 2: Limiting Expressions for the Bayes Estimators as $\mathrm{t} \rightarrow \infty$ Using the UP and the JP under PLF

\begin{tabular}{|c|c|c|}
\hline Parameters & $\begin{array}{l}\text { Bayes Estimators } \\
\text { UP }\end{array}$ & JP \\
\hline$\theta_{1}$ & $\frac{\left[\left(\mathrm{n}_{1}+1\right)\left(\mathrm{n}_{1}+2\right)\right]^{1 / 2}}{\sum_{\mathrm{k}=1}^{\mathrm{n}_{1}} \mathrm{x}_{1 \mathrm{k}}^{-2}}$ & $\frac{\left[\mathrm{n}_{1}\left(\mathrm{n}_{1}+1\right)\right]^{1 / 2}}{\sum_{\mathrm{k}=1}^{\mathrm{n}_{1}} \mathrm{x}_{1 \mathrm{k}}^{-2}}$ \\
\hline$\theta_{2}$ & $\frac{\left[\left(\mathrm{n}_{2}+1\right)\left(\mathrm{n}_{2}+2\right)\right]^{1 / 2}}{\sum_{\mathrm{k}=1}^{\mathrm{n}_{2}} \mathrm{x}_{2 \mathrm{k}}^{-2}}$ & $\frac{\left[\mathrm{n}_{2}\left(\mathrm{n}_{2}+2\right)\right]^{1 / 2}}{\sum_{\mathrm{k}=1}^{\mathrm{n}_{2}} \mathrm{x}_{2 \mathrm{k}}^{-2}}$ \\
\hline$\theta_{3}$ & $\frac{\left[\left(\mathrm{n}_{3}+1\right)\left(\mathrm{n}_{3}+2\right)\right]^{1 / 2}}{\sum_{\mathrm{k}=1}^{\mathrm{n}_{3}} \mathrm{x}_{3 \mathrm{k}}^{-2}}$ & $\frac{\left[\mathrm{n}_{3}\left(\mathrm{n}_{3}+2\right)\right]^{1 / 2}}{\sum_{\mathrm{k}=1}^{\mathrm{n}_{3}} \mathrm{x}_{3 \mathrm{k}}^{-2}}$ \\
\hline $\mathrm{p}_{1}$ & {$\left[\frac{\left(\mathrm{n}_{1}+1\right)\left(\mathrm{n}_{1}+2\right)}{(\mathrm{n}+4)}\right]^{1 / 2}$} & {$\left[\frac{\left(\mathrm{n}_{1}+1\right)\left(\mathrm{n}_{1}+2\right)}{(\mathrm{n}+4)}\right]^{1 / 2}$} \\
\hline $\mathrm{p}_{2}$ & {$\left[\frac{\left(\mathrm{n}_{2}+1\right)\left(\mathrm{n}_{2}+2\right)}{(\mathrm{n}+4)}\right]^{1 / 2}$} & {$\left[\frac{\left(\mathrm{n}_{2}+1\right)\left(\mathrm{n}_{2}+2\right)}{(\mathrm{n}+4)}\right]^{1 / 2}$} \\
\hline
\end{tabular}

Table 3: Limiting Expressions for the Bayes Estimators as $t \rightarrow \infty$ Using the GP and the EP under PLF

\begin{tabular}{|c|c|c|}
\hline Parameters & $\begin{array}{l}\text { Bayes Estimators } \\
\text { Gamma Prior }\end{array}$ & Exponential Prior \\
\hline$\theta_{1}$ & $\frac{\left[\left(\mathrm{n}_{1}+\mathrm{a}_{1}\right)\left(\mathrm{n}_{1}+\mathrm{a}_{1}+1\right)\right]^{1 / 2}}{\sum_{\mathrm{k}=1}^{\mathrm{n}_{1}} \mathrm{x}_{1 \mathrm{k}}^{-2}+\mathrm{b}_{1}}$ & $\frac{\left[\left(\mathrm{n}_{1}+1\right)\left(\mathrm{n}_{1}+2\right)\right]^{1 / 2}}{\sum_{\mathrm{k}=1}^{\mathrm{n}_{1}} \mathrm{x}_{1 \mathrm{k}}^{-2}+\mathrm{w}_{1}}$ \\
\hline$\theta_{2}$ & $\frac{\left[\left(\mathrm{n}_{2}+\mathrm{a}_{2}\right)\left(\mathrm{n}_{2}+\mathrm{a}_{2}+1\right)\right]^{1 / 2}}{\sum_{\mathrm{k}=1}^{\mathrm{n}_{2}} \mathrm{x}_{2 \mathrm{k}}^{-2}+\mathrm{b}_{2}}$ & $\frac{\left[\left(\mathrm{n}_{2}+1\right)\left(\mathrm{n}_{2}+2\right)\right]^{1 / 2}}{\sum_{\mathrm{k}=1}^{\mathrm{n}_{2}} \mathrm{x}_{2 \mathrm{k}}^{-2}+\mathrm{w}_{2}}$ \\
\hline$\theta_{3}$ & $\frac{\left[\left(\mathrm{n}_{3}+\mathrm{a}_{3}\right)\left(\mathrm{n}_{3}+\mathrm{a}_{3}+1\right)\right]^{1 / 2}}{\sum_{\mathrm{k}=1}^{\mathrm{n}_{3}} \mathrm{x}_{3 \mathrm{k}}^{-2}+\mathrm{b}_{3}}$ & $\frac{\left[\left(\mathrm{n}_{3}+1\right)\left(\mathrm{n}_{3}+2\right)\right]^{1 / 2}}{\sum_{\mathrm{k}=1}^{\mathrm{n}_{3}} \mathrm{x}_{3 \mathrm{k}}^{-2}+\mathrm{w}_{3}}$ \\
\hline $\mathrm{p}_{1}$ & {$\left[\frac{\left(\mathrm{n}_{1}+\mathrm{a}\right)\left(\mathrm{n}_{1}+\mathrm{a}+1\right)}{(\mathrm{n}+\mathrm{a}+\mathrm{b}+\mathrm{c})(\mathrm{n}+\mathrm{a}+\mathrm{b}+\mathrm{c}+1)}\right.$} & {$\left[\frac{\left(\mathrm{n}_{1}+\mathrm{a}\right)\left(\mathrm{n}_{1}+\mathrm{a}+1\right)}{(\mathrm{n}+\mathrm{a}+\mathrm{b}+\mathrm{c})(\mathrm{n}+\mathrm{a}+\mathrm{b}+\mathrm{c}+1)}\right.$} \\
\hline $\mathrm{p}_{2}$ & {$\left[\frac{\left(\mathrm{n}_{2}+\mathrm{b}\right)\left(\mathrm{n}_{2}+\mathrm{b}+1\right)}{(\mathrm{n}+\mathrm{a}+\mathrm{b}+\mathrm{c})(\mathrm{n}+\mathrm{a}+\mathrm{b}+\mathrm{c}+1)}\right.$} & {$\left[\frac{\left(\mathrm{n}_{2}+\mathrm{b}\right)\left(\mathrm{n}_{2}+\mathrm{b}+1\right)}{(\mathrm{n}+\mathrm{a}+\mathrm{b}+\mathrm{c})(\mathrm{n}+\mathrm{a}+\mathrm{b}+\mathrm{c}+1)}\right.$} \\
\hline
\end{tabular}

Table 4: Limiting Expressions for the Bayes Estimators as $\mathrm{t} \rightarrow \infty$ Using the UP and the JP, GP and EP under DLF

\begin{tabular}{|c|c|c|c|c|}
\hline Parameters & $\begin{array}{l}\text { Bayes Est } \\
\text { UP }\end{array}$ & JP & Gamma prior & Exponential Prior \\
\hline \multirow[b]{2}{*}{$\theta_{1}$} & $\mathrm{n}_{1}+2$ & $\mathrm{n}_{1}$ & $\mathrm{n}_{1}+\mathrm{a}_{1}+1$ & $\mathrm{n}_{1}+2$ \\
\hline & $\overline{\sum_{k=1}^{n} x_{1 k}^{-2}}$ & $\overline{\sum_{\mathrm{k}=1}^{\mathrm{n}_{1}} \mathrm{x}_{1 \mathrm{k}}^{-2}}$ & $\overline{\sum_{k=1}^{n_{1}} x_{1 k}^{-2}+b_{1}}$ & $\left(\sum_{\mathrm{k}=1}^{\mathrm{n}_{1}} \mathrm{x}_{1 \mathrm{k}}^{-2}+\mathrm{w}_{1}\right.$ \\
\hline \multirow[b]{2}{*}{$\theta_{2}$} & $\mathrm{n}_{2}+2$ & $\mathrm{n}_{2}$ & $\mathrm{n}_{2}+\mathrm{a}_{2}+1$ & $\mathrm{n}_{2}+2$ \\
\hline & $\sum_{k=1}^{n_{2}} x_{2 k}^{-2}$ & $\sum_{k=1}^{n_{2}} x_{2 k}^{-2}$ & $\overline{\sum_{k=1}^{n}} x_{2 k}^{-2}+b_{2}$ & $\left(\sum_{k=1}^{n_{2}} x_{2 k}^{-2}+w_{2}\right.$ \\
\hline \multirow[b]{2}{*}{$\theta_{3}$} & $\mathrm{n}_{3}+2$ & $\mathrm{n}_{3}$ & $\mathrm{n}_{3}+\mathrm{a}_{3}+1$ & $\mathrm{n}_{3}+2$ \\
\hline & $\overline{\sum_{k=1}^{n} x_{3 k}^{-2}}$ & $\overline{\sum_{k=1}^{n} x_{3 k}^{-2}}$ & $\overline{\sum_{k=1}^{n_{3}} x_{3 k}^{-2}+b_{3}}$ & $\left(\sum_{k=1}^{n_{3}} x_{3 k}^{-2}+w_{3}\right)$ \\
\hline \multirow{2}{*}{$\mathrm{p}_{1}$} & $\mathrm{n}_{1}+2$ & $\underline{\mathrm{n}_{1}+2}$ & $\mathrm{n}_{1}+\mathrm{a}+1$ & $\mathrm{n}_{1}+\mathrm{a}+1$ \\
\hline & $\overline{n+4}$ & $n+4$ & $\overline{\mathrm{n}+\mathrm{a}+\mathrm{b}+\mathrm{c}}$ & $\overline{n+a+b+c}$ \\
\hline $\mathrm{p}_{2}$ & $\overline{n+4}$ & $n+4$ & $\overline{n+a+b+c}$ & $\overline{n+a+b+c}$ \\
\hline
\end{tabular}


Table 5: Limiting Expressions for the Posterior Risks as $t \rightarrow \infty$ Using the UP and the JP, GP and EP under SELF

\begin{tabular}{|c|c|c|c|c|}
\hline Parameters & UP & JP & Gamma prior & $\begin{array}{l}\text { Exponential } \\
\text { Prior }\end{array}$ \\
\hline \multirow[b]{2}{*}{$\theta_{1}$} & $\mathrm{n}_{1}+1$ & $\mathrm{n}_{1}$ & $\mathrm{n}_{1}+\mathrm{a}_{1}$ & $\mathrm{n}_{1}+1$ \\
\hline & $\overline{\left(\sum_{k=1}^{n_{1}} x_{1 k}^{-2}\right)^{2}}$ & $\overline{\left(\sum_{k=1}^{n_{1}} x_{1 k}^{-2}\right)^{2}}$ & $\overline{\left(\sum_{k=1}^{n_{1}} x_{1 k}^{-2}+b_{1}\right)^{2}}$ & $\overline{\left(\sum_{\mathrm{k}=1}^{\mathrm{n}_{1}} \mathrm{x}_{1 \mathrm{k}}^{-2}+\mathrm{w}_{1}\right)^{2}}$ \\
\hline \multirow[b]{2}{*}{$\theta_{2}$} & $\mathrm{n}_{2}+1$ & $\mathrm{n}_{2}$ & $\mathrm{n}_{2}+\mathrm{a}_{2}$ & $\mathrm{n}_{2}+1$ \\
\hline & $\overline{\left(\sum_{k=1}^{n} x_{2 k}^{-2}\right)^{2}}$ & $\overline{\left(\sum_{k=1}^{n_{2}} x_{2 k}^{-2}\right)^{2}}$ & $\left(\sum_{\mathrm{k}=1}^{\mathrm{n}_{2}} \mathrm{x}_{2 \mathrm{k}}^{-2}+\mathrm{b}_{2}\right)^{2}$ & $\overline{\left(\sum_{k=1}^{n_{2}} x_{2 k}^{-2}+w_{2}\right)^{2}}$ \\
\hline \multirow[b]{2}{*}{$\theta_{3}$} & $\mathrm{n}_{3}+1$ & $\mathrm{n}_{3}$ & $\mathrm{n}_{3}+\mathrm{a}_{3}$ & $\mathrm{n}_{3}+1$ \\
\hline & $\overline{\left(\sum_{k=1}^{n} x_{3 k}^{-2}\right)^{2}}$ & $\overline{\left(\sum_{k=1}^{n} x_{3 k}^{-2}\right)^{2}}$ & $\left.\overline{\left(\sum_{\mathrm{k}=1}^{\mathrm{n}_{3}} \mathrm{x}_{3 \mathrm{k}}-2\right.}+\mathrm{b}_{3}\right)^{2}$ & $\overline{\left(\sum_{k=1}^{n} x_{3 k}^{-2}+w_{3}\right)^{2}}$ \\
\hline \multirow[b]{2}{*}{$\mathrm{p}_{1}$} & $\left(\mathrm{n}_{1}+1\right)\left(\mathrm{n}_{2}+\mathrm{n}_{3}+2\right)$ & $\left(\mathrm{n}_{1}+1\right)\left(\mathrm{n}_{2}+\mathrm{n}_{3}+2\right)$ & $\left(\mathrm{n}_{1}+\mathrm{a}\right)\left(\mathrm{n}_{2}+\mathrm{n}_{3}+\mathrm{b}+\mathrm{c}\right)$ & $\left(\mathrm{n}_{1}+\mathrm{a}\right)\left(\mathrm{n}_{2}+\mathrm{n}_{3}+\mathrm{b}+\mathrm{c}\right)$ \\
\hline & $(\mathrm{n}+3)^{2}(\mathrm{n}+4)$ & $(\mathrm{n}+3)^{2}(\mathrm{n}+4)$ & $(\mathrm{n}+\mathrm{a}+\mathrm{b}+\mathrm{c})^{2}(\mathrm{n}+\mathrm{a}+\mathrm{b}+\mathrm{c}+1)$ & $(\mathrm{n}+\mathrm{a}+\mathrm{b}+\mathrm{c})^{2}(\mathrm{n}+\mathrm{a}+\mathrm{b}+\mathrm{c}+1)$ \\
\hline \multirow[b]{2}{*}{$\mathrm{p}_{2}$} & $\left(n_{2}+1\right)\left(n_{1}+n_{3}+2\right)$ & $\left(n_{2}+1\right)\left(n_{1}+n_{3}+2\right)$ & $\left(\mathrm{n}_{2}+\mathrm{b}\right)\left(\mathrm{n}_{1}+\mathrm{n}_{3}+\mathrm{b}+\mathrm{c}\right)$ & $\left(\mathrm{n}_{2}+\mathrm{b}\right)\left(\mathrm{n}_{1}+\mathrm{n}_{3}+\mathrm{b}+\mathrm{c}\right)$ \\
\hline & $(n+3)^{2}(n+4)$ & $(n+3)^{2}(n+4)$ & $(\mathrm{n}+\mathrm{a}+\mathrm{b}+\mathrm{c})^{2}(\mathrm{n}+\mathrm{a}+\mathrm{b}+\mathrm{c}+1)$ & $(n+a+b+c)^{2}(n+a+b+c+1)$ \\
\hline
\end{tabular}

Table 6: Limiting Expressions for the Posterior Risks as $t \rightarrow \infty$ Using the UP and the JP, GP and EP under PLF

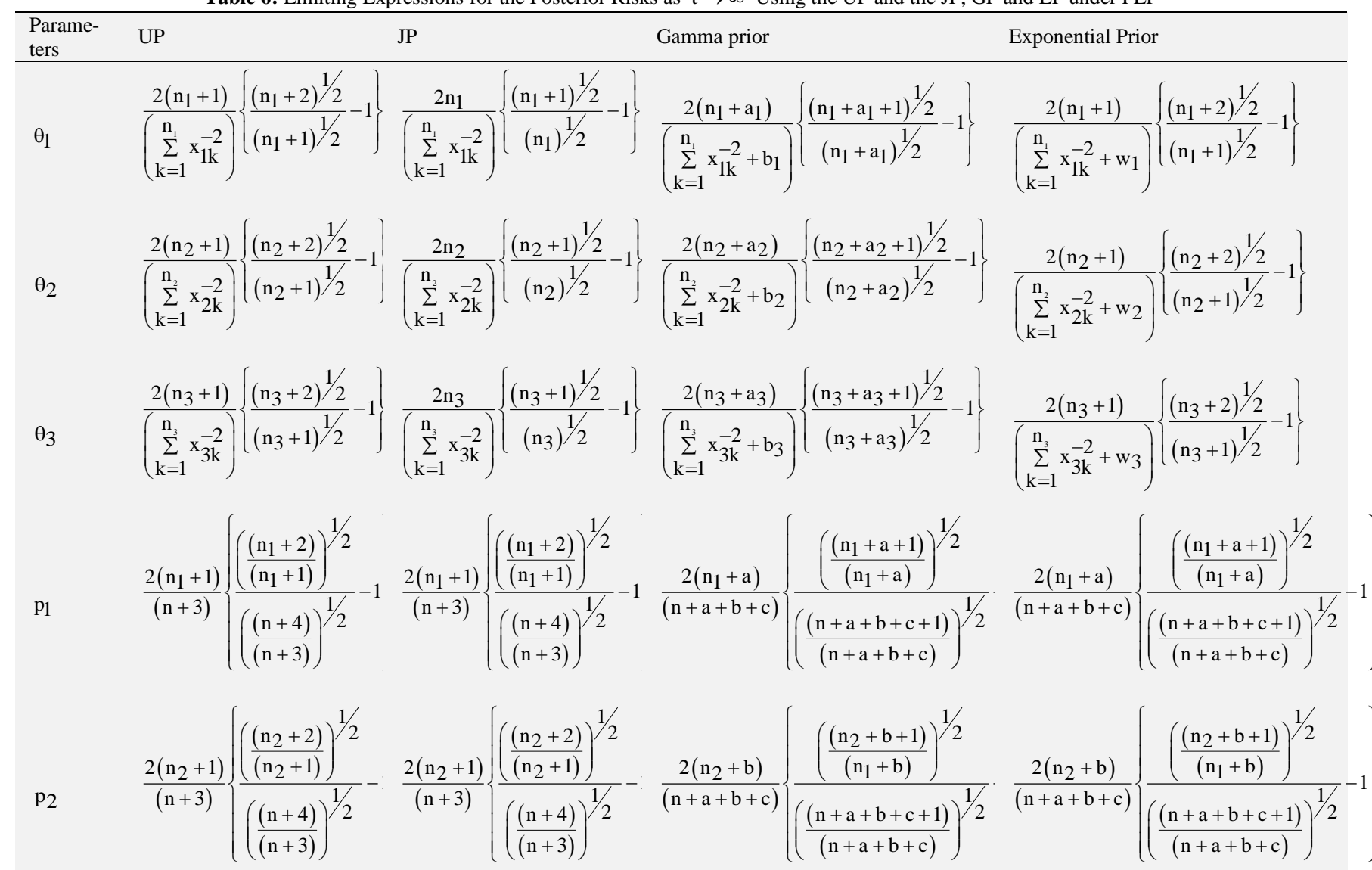

Table 7: Limiting Expressions for the Posterior Risks as $\mathrm{t} \rightarrow \infty$ Using the UP and the JP, GP and EP under DLF

\begin{tabular}{|c|c|c|c|c|}
\hline Parameters & $\begin{array}{l}\text { Posterior Risks } \\
\text { UP }\end{array}$ & JP & Gamma prior & Exponential Prior \\
\hline \multirow{2}{*}{$\theta_{1}$} & 1 & 1 & 1 & 1 \\
\hline & $\overline{n_{1}+2}$ & $\overline{n_{1}+1}$ & $\overline{n_{1}+a_{1}+1}$ & $\overline{n_{1}+2}$ \\
\hline \multirow{2}{*}{$\theta_{2}$} & 1 & 1 & 1 & 1 \\
\hline & $\mathrm{n}_{2}+2$ & $\mathrm{n}_{2}+1$ & $\mathrm{n}_{2}+\mathrm{a}_{2}+1$ & $\mathrm{n}_{2}+2$ \\
\hline \multirow{2}{*}{$\theta_{3}$} & 1 & 1 & 1 & 1 \\
\hline & $\mathrm{n}_{3}+2$ & $\overline{n_{3}+1}$ & $\mathrm{n}_{3}+\mathrm{a}_{3}+1$ & $\mathrm{n}_{3}+2$ \\
\hline \multirow{2}{*}{$\mathrm{p}_{1}$} & $\left(\mathrm{n}_{2}+\mathrm{n}_{3}+2\right)$ & $\left(\mathrm{n}_{2}+\mathrm{n}_{3}+2\right)$ & $\left(\mathrm{n}_{2}+\mathrm{n}_{3}+\mathrm{b}+\mathrm{c}\right)$ & $\left(\mathrm{n}_{2}+\mathrm{n}_{3}+\mathrm{b}+\mathrm{c}\right)$ \\
\hline & $\overline{\left(n_{1}+2\right)(n+3)}$ & $\overline{\left(n_{1}+2\right)(n+3)}$ & $\overline{\left(n_{1}+a+1\right)(n+a+b+c)}$ & $\overline{\left(n_{1}+a+1\right)(n+a+b+c)}$ \\
\hline
\end{tabular}




$\mathrm{p}_{2} \frac{\left(\mathrm{n}_{1}+\mathrm{n}_{3}+2\right)}{\left(\mathrm{n}_{2}+2\right)(\mathrm{n}+3)} \quad \frac{\left(\mathrm{n}_{1}+\mathrm{n}_{3}+2\right)}{\left(\mathrm{n}_{2}+2\right)(\mathrm{n}+3)} \quad \frac{\left(\mathrm{n}_{1}+\mathrm{n}_{3}+\mathrm{a}+\mathrm{c}\right)}{\left(\mathrm{n}_{2}+\mathrm{b}+1\right)(\mathrm{n}+\mathrm{a}+\mathrm{b}+\mathrm{c})} \quad \frac{\left(\mathrm{n}_{1}+\mathrm{n}_{3}+\mathrm{a}+\mathrm{c}\right)}{\left(\mathrm{n}_{2}+\mathrm{b}+1\right)(\mathrm{n}+\mathrm{a}+\mathrm{b}+\mathrm{c})}$

\section{Simulation study}

A thorough simulation study was conducted in order to investigate the performance of the Bayes estimators, impact of sample size and censoring rate in the fit of the model. Samples of sizes $n=25$, $50,100,200$ are generated from a 3-component mixture of the inverse Rayleigh distributions with different set of the parametric values $\theta_{1}, \theta_{2}, \theta_{3}, \mathrm{p}_{1} \& \mathrm{p}_{2}$ fixed as $\left(\theta_{1}, \theta_{2}, \theta_{3}, \mathrm{p}_{1}, \mathrm{p}_{2}\right)=(0.25,0.50$ $0.75,0.20,0.65),(0.75,0.50,0.25,0.65,0.20),(0.50,0.50,0.50$, $0.40,0.40)$.For fixed sample size, test termination time and set of parameters, the simulation is repeated 1000 times and the results are then averaged. Sample of sizes $p_{1} n, p_{2} n$ and $\left(1-p_{1}-p_{2}\right) n$ are chosen randomly from first component density $\mathrm{f}_{1}\left(\mathrm{x} ; \theta_{1}\right)$, second component density $f_{2}\left(x ; \theta_{2}\right)$ and third component density $f_{3}\left(x ; \theta_{3}\right)$, respectively. The observations which are greater than a fixed $t$ are declared as censored observations. For each $t$ only failures are identified either as a member of subpopulation-I or subpopulation-II or subpopulation-III. Based on each sample size, the Bayes estimators (BEs) and Posterior risks are computed using the non-informative and informative priors (IP) under SELF, PLF and DLF. In order to evaluate the impact of test termination time on Bayes estimators, the Type-I right censoring scheme is used for fixed test termination time $\mathrm{t}=15$ and 20 . For For each of the 1000 samples, the Bayes estimators and Posterior risks were computed using a routine in Mathematica 10.0 and the results are presented in Table 8-19 given below. The simulation study (appendix) provides us some interesting properties of the Bayes estimates. The properties are highlighted in terms of sample sizes, size of mixing proportion parameters, different loss functions and censoring rates. It is observed that due to censoring, the posterior risks of all the parameters are reduced with an increase in sample size.

It is also observed that Posterior risks using the informative priors (IP) are smaller than the Posterior risks using the UP and the JP for different sample sizes and test termination times. Also, the Posterior risks using the JP are smaller than using the UP for different sample sizes and test termination times. It is also observed that in estimating the component parameters $\theta_{1}, \theta_{2} \& \theta_{3}$ and Posterior risks are smaller under DLF than under SELF and PLF at different sample sizes and test termination times considered in the study. However, for estimating the mixing proportions, SELF yields smaller Posterior risks than PLF and DLF, at different sample sizes and test termination times. Thus, DLF is more suitable for estimating component parameters and SELF is preferable choice for estimating the proportion parameters.

Table 8: Bayes Estimates (BEs) and Posterior Risks (PRs) of 3-component Mixture of Inverse Rayleigh Distributions Using the UP under SELF, PLF and DLF with $\theta_{1}=0.25, \theta_{2}=0.50, \theta_{3}=0.75, \mathrm{p}_{1}=0.20, \mathrm{p}_{2}=0.65$ and $\mathrm{t}=15,20$

\begin{tabular}{|c|c|c|c|c|c|c|c|c|}
\hline $\mathrm{t}$ & $\mathrm{n}$ & \multicolumn{2}{|c|}{ Loss Functions } & $\begin{array}{l}\text { UP } \\
\hat{\theta}_{1}\end{array}$ & $\hat{\theta}_{2}$ & $\hat{\theta}_{3}$ & $\hat{\mathrm{p}}_{1}$ & $\hat{\mathrm{p}}_{2}$ \\
\hline \multirow{22}{*}{15} & \multirow{6}{*}{25} & \multirow{2}{*}{ SELF } & $\mathrm{BE}$ & 0.36999 & 0.55842 & 1.24979 & 0.21437 & 0.60670 \\
\hline & & & PR & 0.30547 & 0.019635 & 0.494425 & 0.005815 & 0.008244 \\
\hline & & \multirow{2}{*}{ PLF } & $\mathrm{BE}$ & 0.40052 & 0.58594 & 1.37124 & 0.22742 & 0.61358 \\
\hline & & & PR & 0.059500 & 0.033061 & 0.23896 & 0.026320 & 0.013509 \\
\hline & & \multirow{2}{*}{ DLF } & $\mathrm{BE}$ & 0.43951 & 0.59933 & 1.49256 & 0.24147 & 0.62040 \\
\hline & & & PR & 0.142912 & 0.055616 & 0.166733 & 0.112302 & 0.011133 \\
\hline & \multirow{6}{*}{50} & \multirow{2}{*}{ SELF } & $\mathrm{BE}$ & 0.30588 & 0.52882 & 0.97946 & 0.20760 & 0.62252 \\
\hline & & & PR & 0.009512 & 0.008756 & 0.125443 & 0.003050 & 0.004359 \\
\hline & & \multirow{2}{*}{ PLF } & $\mathrm{BE}$ & 0.31619 & 0.54671 & 1.00714 & 0.01474 & 0.62610 \\
\hline & & & PR & 0.026952 & 0.016213 & 0.103557 & 0.014442 & 0.006982 \\
\hline & & \multirow{2}{*}{ DLF } & $\mathrm{BE}$ & 0.33304 & 0.54652 & 1.0667 & 0.22236 & 0.62946 \\
\hline & & & PR & 0.083379 & 0.029445 & 0.100213 & 0.066085 & 0.111326 \\
\hline & \multirow{6}{*}{100} & \multirow{2}{*}{ SELF } & $\mathrm{BE}$ & 0.27924 & 0.51788 & 0.85678 & 0.20391 & 0.64076 \\
\hline & & & PR & 0.003934 & 0.004129 & 0.049580 & 0.001563 & 0.002218 \\
\hline & & \multirow{2}{*}{ PLF } & $\mathrm{BE}$ & 0.28595 & 0.51917 & 0.88042 & 0.20775 & 0.64238 \\
\hline & & & PR & 0.013158 & 0.007785 & 0.052689 & 0.007594 & 0.003458 \\
\hline & & \multirow{2}{*}{ DLF } & $\mathrm{BE}$ & 0.29101 & 0.51902 & 0.90183 & 0.21157 & 0.64416 \\
\hline & & & PR & 0.045492 & 0.014938 & 0.058942 & 0.036225 & 0.005374 \\
\hline & \multirow{6}{*}{200} & \multirow{2}{*}{ SELF } & $\mathrm{BE}$ & 0.26387 & 0.50864 & 0.79626 & 0.20201 & 0.64531 \\
\hline & & & PR & 0.001746 & 0.001993 & 0.021177 & 0.000791 & 0.001124 \\
\hline & & \multirow{2}{*}{ PLF } & $\mathrm{BE}$ & 0.26981 & 0.50931 & 0.80425 & 0.20398 & 0.64608 \\
\hline & & & PR & 0.006468 & 0.003869 & 0.025390 & 0.003898 & 0.001742 \\
\hline \multirow{21}{*}{20} & & \multirow{2}{*}{ DLF } & $\mathrm{BE}$ & 0.26756 & 0.51363 & 0.82888 & 0.20591 & 0.64701 \\
\hline & & & PR & 0.023830 & 0.007582 & 0.031322 & 0.019021 & 0.002693 \\
\hline & & \multirow{2}{*}{ SELF } & $\mathrm{BE}$ & 0.37718 & 0.57244 & 0.12547 & 0.21431 & 0.60720 \\
\hline & & & PR & 0.032674 & 0.020692 & 0.451357 & 0.005812 & 0.008236 \\
\hline & & & $\mathrm{BE}$ & 0.40654 & 0.57876 & 1.36121 & 0.22738 & 0.61381 \\
\hline & 25 & PLF & PR & 0.060386 & 0.032632 & 0.237261 & 0.026308 & 0.013489 \\
\hline & & & $\mathrm{BE}$ & 0.43914 & 0.60399 & 1.46604 & 0.24405 & 0.62066 \\
\hline & & DLF & PR & 0.14294 & 0.055588 & 0.166887 & 0.112331 & 0.021859 \\
\hline & & SEI F & $\mathrm{BE}$ & 0.30138 & 0.52995 & 0.97660 & 0.20755 & 0.62248 \\
\hline & & SELF & PR & 0.009266 & 0.008782 & 0.1226 & 0.003048 & 0.004357 \\
\hline & 50 & PI F & $\mathrm{BE}$ & 0.31873 & 0.54206 & 1.025 & 0.21476 & 0.62602 \\
\hline & 50 & PLF & PR & 0.027152 & 0.016072 & 0.105259 & 0.014434 & 0.006979 \\
\hline & & & $\mathrm{BE}$ & 0.32664 & 0.55207 & 1.08674 & 0.22224 & 0.62963 \\
\hline & & DLF & PR & 0.083373 & 0.029424 & 0.100125 & 0.066078 & 0.011111 \\
\hline & & & $\mathrm{BE}$ & 0.27636 & 0.51432 & 0.86003 & 0.20923 & 0.64073 \\
\hline & & SELF & PR & 0.003826 & 0.004073 & 0.049861 & 0.001562 & 0.002216 \\
\hline & & & $\mathrm{BE}$ & 0.28315 & 0.52107 & 0.88695 & 0.20773 & 0.64243 \\
\hline & 100 & PLF & PR & 0.013023 & 0.007810 & 0.053023 & 0.007588 & 0.003454 \\
\hline & & & $\mathrm{BE}$ & 0.29275 & 0.52194 & 0.9132 & 0.21158 & 0.64412 \\
\hline & & DLF & PR & 0.045473 & 0.014935 & 0.058878 & 0.0366206 & 0.005371 \\
\hline & 200 & SELF & $\mathrm{BE}$ & 0.26457 & 0.50856 & 0.80037 & 0.20196 & 0.64534 \\
\hline
\end{tabular}




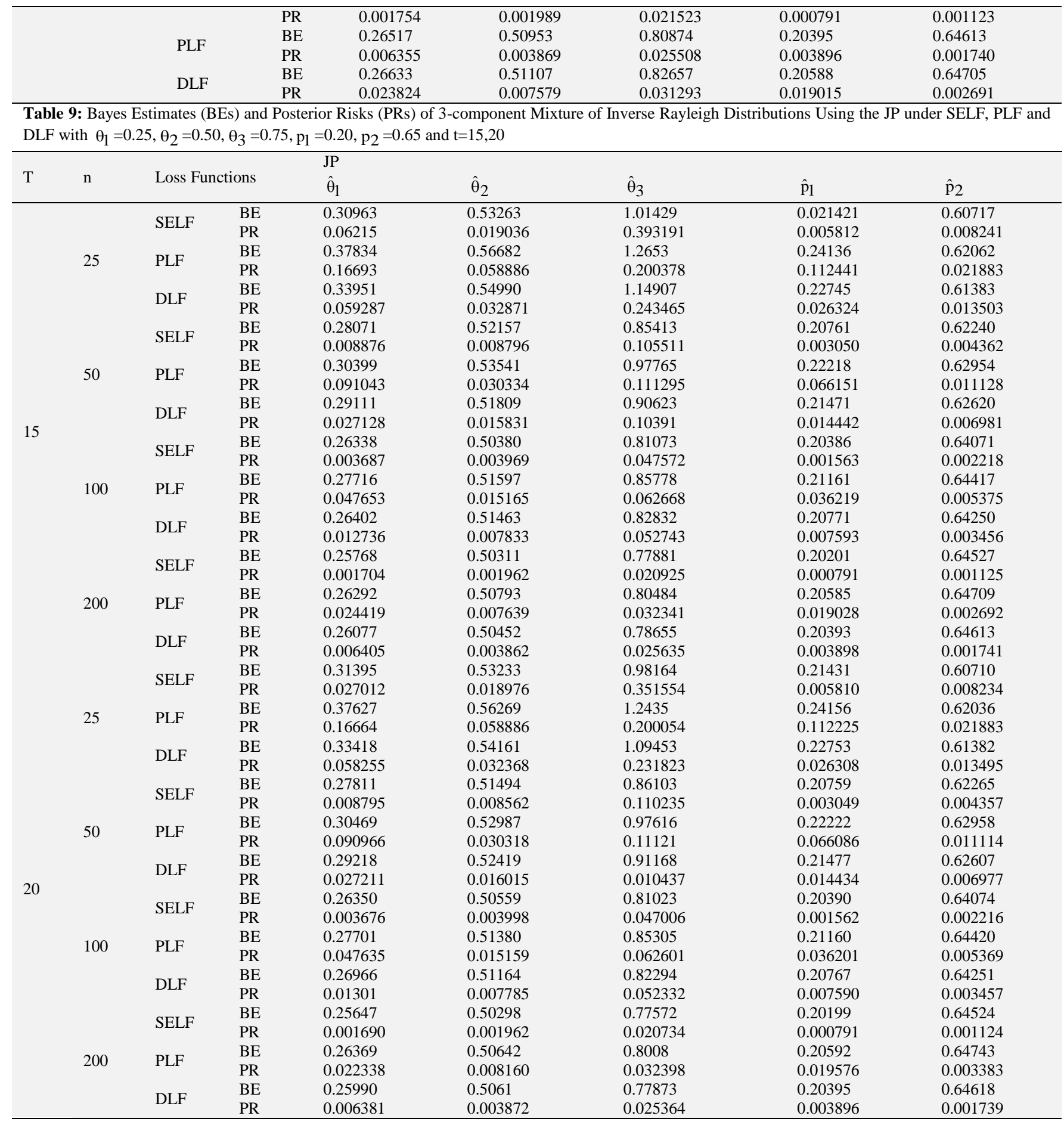

Table 10: Bayes Estimates (BEs) and Posterior Risks (PRs) of 3-component Mixture of Inverse Rayleigh Distributions Using the UP under SELF, PLF and DLF with $\theta_{1}=0.75, \theta_{2}=0.50, \theta_{3}=0.25, \mathrm{p}_{1}=0.65, \mathrm{p}_{2}=0.20$ and $\mathrm{t}=15,20$

\begin{tabular}{|c|c|c|c|c|c|c|c|c|}
\hline $\mathrm{T}$ & $\mathrm{n}$ & \multicolumn{2}{|c|}{ Loss Functions } & $\begin{array}{l}\text { UP } \\
\hat{\theta}_{1}\end{array}$ & $\hat{\theta}_{2}$ & $\hat{\theta}_{3}$ & $\hat{\mathrm{p}}_{1}$ & $\hat{\mathrm{p}}_{2}$ \\
\hline \multirow{15}{*}{15} & \multirow{6}{*}{25} & \multirow{2}{*}{ SELF } & $\mathrm{BE}$ & 0.85579 & 0.73238 & 0.52775 & 0.60699 & 0.21448 \\
\hline & & & PR & 0.406235 & 0.112065 & 0.058728 & 0.008243 & 0.005822 \\
\hline & & \multirow{2}{*}{ PLF } & $\mathrm{BE}$ & 0.86855 & 0.80514 & 0.44510 & 0.61371 & 0.22753 \\
\hline & & & PR & 0.048992 & 0.119603 & 0.077605 & 0.013504 & 0.026338 \\
\hline & & \multirow{2}{*}{ DLF } & $\mathrm{BE}$ & 0.89841 & 0.87655 & 0.50184 & 0.62052 & 0.24160 \\
\hline & & & PR & 0.055616 & 0.142993 & 0.166805 & 0.021888 & 0.112386 \\
\hline & \multirow{6}{*}{50} & \multirow{2}{*}{ SELF } & $\mathrm{BE}$ & 0.81877 & 0.63138 & 0.33623 & 0.63637 & 0.21126 \\
\hline & & & PR & 0.020696 & 0.041191 & 0.016930 & 0.004361 & 0.003145 \\
\hline & & \multirow{2}{*}{ PLF } & $\mathrm{BE}$ & 0.83278 & 0.66050 & 0.35934 & 0.63979 & 0.21952 \\
\hline & & & PR & 0.024302 & 0.056073 & 0.041069 & 0.006836 & 0.014639 \\
\hline & & \multirow{2}{*}{ DLF } & $\mathrm{BE}$ & 0.83440 & 0.68578 & 0.38514 & 0.64312 & 0.22619 \\
\hline & & & PR & 0.028985 & 0.083150 & 0.111127 & 0.010664 & 0.065855 \\
\hline & \multirow{3}{*}{100} & \multirow{2}{*}{ SELF } & $\mathrm{BE}$ & 0.77465 & 0.55633 & 0.28541 & 0.64078 & 0.20388 \\
\hline & & & PR & 0.009245 & 0.015625 & 0.005493 & 0.002218 & 0.001565 \\
\hline & & PLF & $\mathrm{BE}$ & 0.78571 & 0.56710 & 0.29378 & 0.64241 & 0.20775 \\
\hline
\end{tabular}




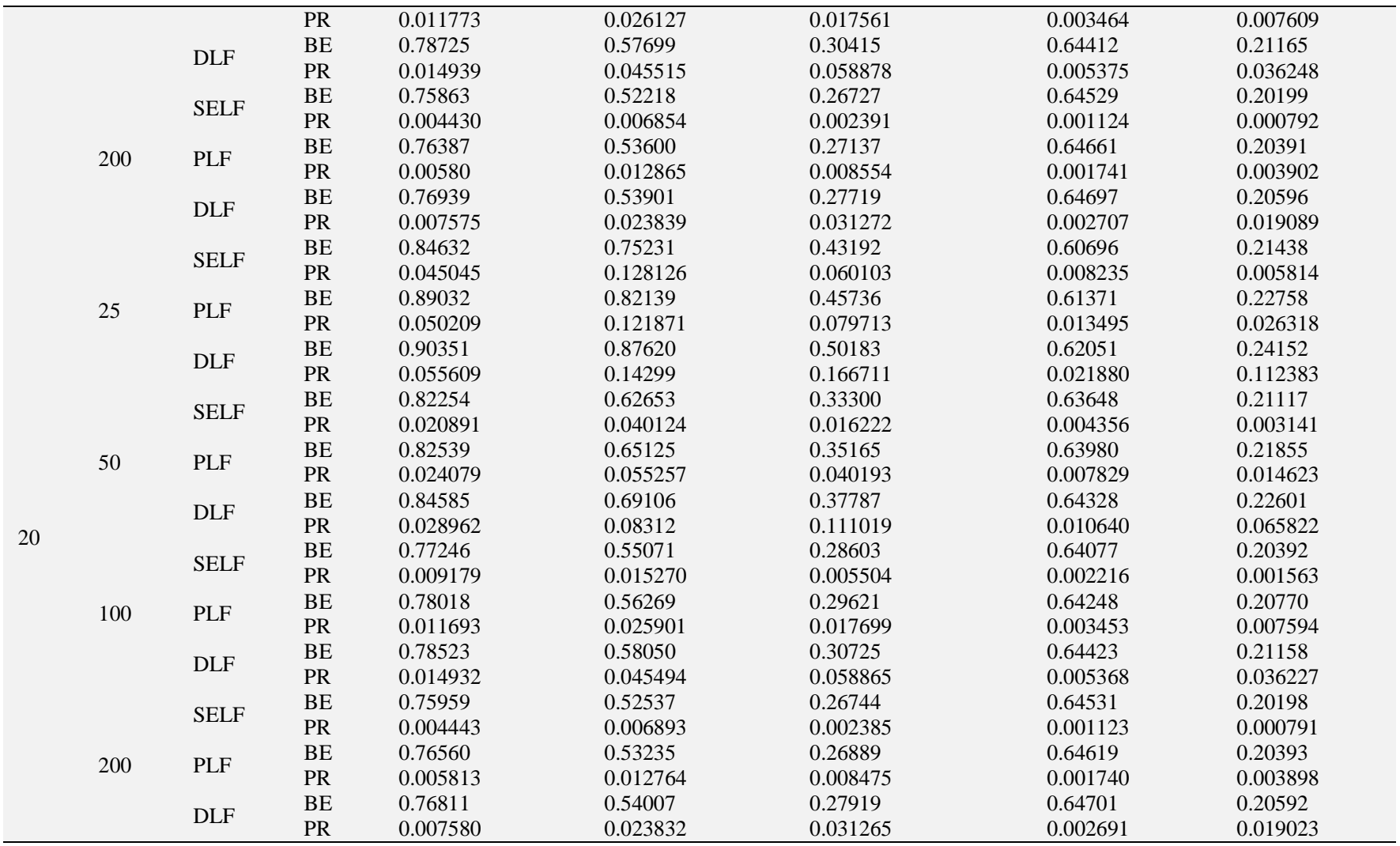

Table 11: Bayes Estimates (BEs) and Posterior Risks (PRs) of 3-component Mixture of Inverse Rayleigh Distributions Using the JP Under SELF, PLF and DLF with $\theta_{1}=0.75, \theta_{2}=0.50, \theta_{3}=0.25, \mathrm{p}_{1}=0.65, \mathrm{p}_{2}=0.20$ and $\mathrm{t}=15,20$

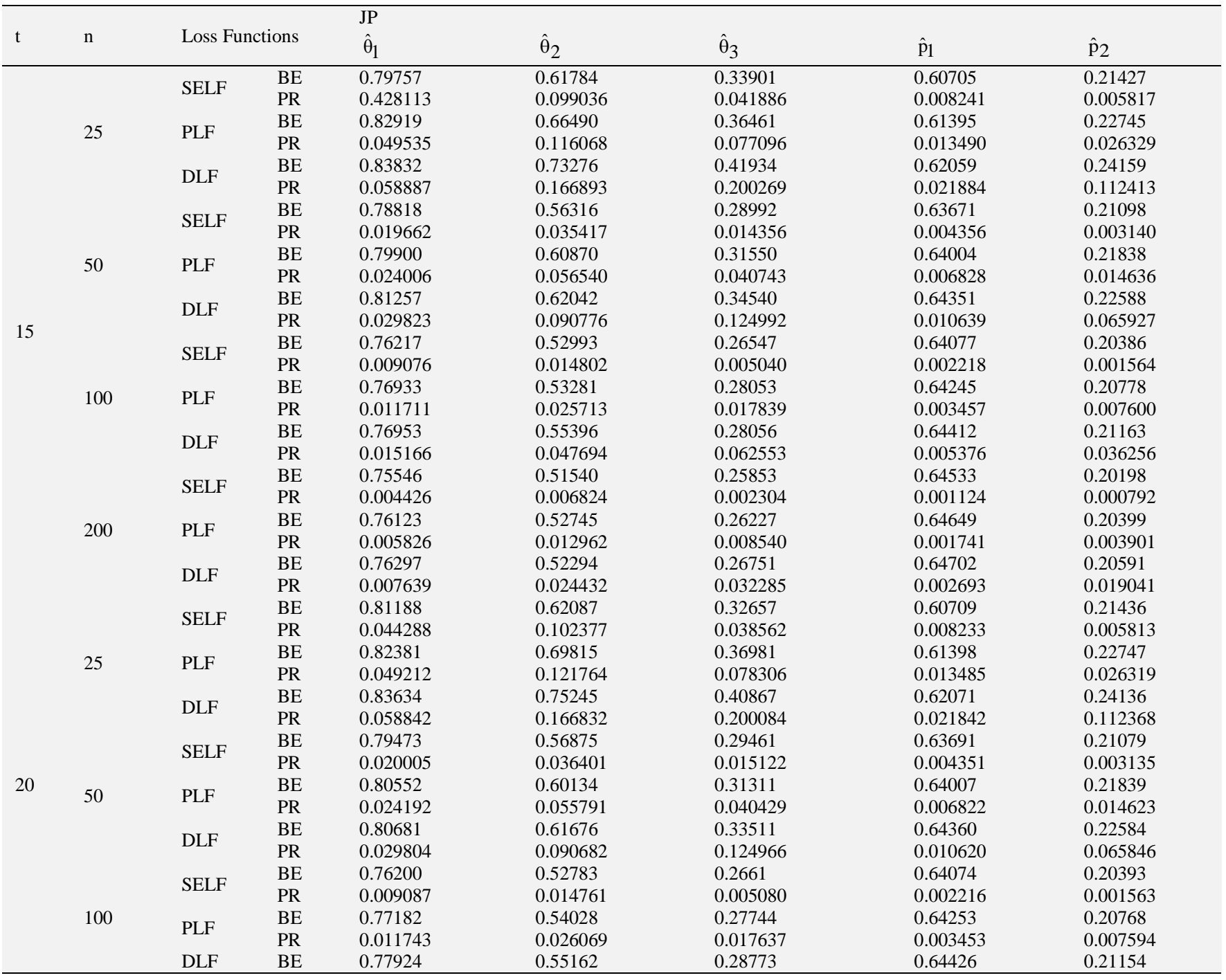




\begin{tabular}{|c|c|c|c|c|c|c|c|}
\hline \multirow{7}{*}{200} & \multirow{3}{*}{ SELF } & PR & 0.015159 & 0.047682 & 0.062555 & 0.005369 & 0.036245 \\
\hline & & $\mathrm{BE}$ & 0.75675 & 0351312 & 0.25800 & 0.64533 & 0.20197 \\
\hline & & PR & 0.004440 & 0.006774 & 0.002299 & 0.001123 & 0.000791 \\
\hline & \multirow{2}{*}{ PLF } & $\mathrm{BE}$ & 0.75775 & 0.52059 & 0.26232 & 0.64624 & 0.20391 \\
\hline & & PR & 0.005797 & 0.012790 & 0.008539 & 0.001739 & 0.003898 \\
\hline & \multirow{2}{*}{ DLF } & $\mathrm{BE}$ & 0.76023 & 0.52633 & 0.26704 & 0.64700 & 0.20590 \\
\hline & & PR & 0.007637 & 0.024413 & 0.032268 & 0.002691 & 0.019023 \\
\hline
\end{tabular}

Table 12: Bayes Estimates (BEs) and Posterior Risks (PRs) of 3-component Mixture of Inverse Rayleigh Distributions Using the UP under SELF, PLF and DLF with $\theta_{1}=0.50, \theta_{2}=0.50, \theta_{3}=0.50, \mathrm{p}_{1}=0.40, \mathrm{p}_{2}=0.40$ and $\mathrm{t}=15,20$

\begin{tabular}{|c|c|c|c|c|c|c|c|c|}
\hline $\mathrm{t}$ & $\mathrm{n}$ & \multicolumn{2}{|c|}{ Loss Functions } & $\begin{array}{c}\text { UP } \\
\hat{\theta}_{1}\end{array}$ & $\hat{\theta}_{2}$ & $\hat{\theta}_{3}$ & $\hat{\mathrm{p}}_{1}$ & $\hat{\mathrm{p}}_{2}$ \\
\hline \multirow{22}{*}{15} & \multirow{6}{*}{25} & \multirow{2}{*}{ SELF } & $\mathrm{BE}$ & 0.61245 & 0.60466 & 0.74088 & 0.39267 & 0.39294 \\
\hline & & & PR & 0.03900 & 0.037474 & 0.119946 & 0.008241 & 0.008243 \\
\hline & & \multirow{2}{*}{ PLF } & $\mathrm{BE}$ & 0.64979 & 0.63962 & 0.81558 & 0.40309 & 0.40323 \\
\hline & & & PR & 0.055428 & 0.054540 & 0.121156 & 0.020713 & 0.020707 \\
\hline & & \multirow{2}{*}{ DLF } & $\mathrm{BE}$ & 0.66753 & 0.66165 & 0.85625 & 0.41389 & 0.41376 \\
\hline & & & PR & 0.083414 & 0.083448 & 0.143066 & 0.050679 & 0.050714 \\
\hline & \multirow{6}{*}{50} & \multirow{2}{*}{ SELF } & $\mathrm{BE}$ & 0.55239 & 0.55340 & 0.61168 & 0.39619 & 0.39618 \\
\hline & & & PR & 0.015402 & 0.015402 & 0.038175 & 0.004440 & 0.004440 \\
\hline & & \multirow{2}{*}{ PLF } & $\mathrm{BE}$ & 0.56319 & 0.56128 & 0.64479 & 0.40178 & 0.40165 \\
\hline & & & PR & 0.025934 & 0.025849 & 0.054938 & 0.011126 & 0.011128 \\
\hline & & \multirow{2}{*}{ DLF } & $\mathrm{BE}$ & 0.57492 & 0.58277 & 0.65920 & 0.40744 & 0.40742 \\
\hline & & & PR & 0.045513 & 0.045516 & 0.083490 & 0.027504 & 0.027507 \\
\hline & \multirow{6}{*}{100} & \multirow{2}{*}{ SELF } & $\mathrm{BE}$ & 0.52296 & 0.52524 & 0.54667 & 0.39799 & 0.39808 \\
\hline & & & PR & 0.006848 & 0.006913 & 0.015003 & 0.002308 & 0.002308 \\
\hline & & \multirow{2}{*}{ PLF } & $\mathrm{BE}$ & 0.53250 & 0.53182 & 0.56370 & 0.40097 & 0.40097 \\
\hline & & & PR & 0.012771 & 0.012754 & 0.025973 & 0.005779 & 0.005779 \\
\hline & & \multirow{2}{*}{ DLF } & $\mathrm{BE}$ & 0.54348 & 0.53928 & 0.58453 & 0.40379 & 0.40398 \\
\hline & & & PR & 0.023850 & 0.023839 & 0.045562 & 0.014374 & 0.014362 \\
\hline & \multirow{6}{*}{200} & \multirow{2}{*}{ SELF } & $\mathrm{BE}$ & 0.51282 & 0.51582 & 0.52255 & 0.39901 & 0.39890 \\
\hline & & & PR & 0.003294 & 0.003335 & 0.006842 & 0.001178 & 0.001178 \\
\hline & & \multirow{2}{*}{ PLF } & $\mathrm{BE}$ & 0.51640 & 0.51630 & 0.52982 & 0.4004 & 0.40048 \\
\hline & & & PR & 0.006327 & 0.006325 & 0.012712 & 0.002948 & 0.002948 \\
\hline & & DIF & $\mathrm{BE}$ & 0.51501 & 0.51951 & 0.53939 & 0.40203 & 0.40193 \\
\hline & & DLF & PR & 0.012207 & 0.012211 & 0.023849 & 0.007342 & 0.007345 \\
\hline & & & $\mathrm{BE}$ & 0.60417 & 0.60427 & 0.72072 & 0.39283 & 0.39276 \\
\hline & & SELF & PR & 0.037499 & 0.036972 & 0.11148 & 0.008235 & 0.008235 \\
\hline & 25 & & $\mathrm{BE}$ & 0.62746 & 0.64000 & 0.85174 & 0.40339 & 0.40293 \\
\hline & 25 & PLF & PR & 0.053446 & 0.054572 & 0.126424 & 0.020686 & 0.020702 \\
\hline & & & $\mathrm{BE}$ & 0.67759 & 0.65516 & 0.84778 & 0.41387 & 0.41383 \\
\hline & & DLF & PR & 0.083367 & 0.083373 & 0.143013 & 0.050631 & 0.050637 \\
\hline & & & $\mathrm{BE}$ & 0.55542 & 0.55120 & 0.61711 & 0.39611 & 0.39632 \\
\hline & & SELF & PR & 0.015552 & 0.015283 & 0.039071 & 0.004435 & 0.004436 \\
\hline & & & $\mathrm{BE}$ & 0.56801 & 0.56662 & 0.64425 & 0.40186 & 0.40174 \\
\hline & 50 & PLF & PR & 0.026134 & 0.026078 & 0.054935 & 0.011116 & 0.011185 \\
\hline & & & $\mathrm{BE}$ & 0.57743 & 0.58061 & 0.67661 & 0.40740 & 0.40744 \\
\hline & & DLF & PR & 0.045482 & 0.045478 & 0.083402 & 0.027472 & 0.027469 \\
\hline 20 & & SFIF & $\mathrm{BE}$ & 0.52417 & 0.52474 & 0.5566 & 0.39808 & 0.39800 \\
\hline & & SELF & PR & 0.006891 & 0.006910 & 0.015519 & 0.002307 & 0.002306 \\
\hline & 100 & & $\mathrm{BE}$ & 0.53159 & 0.53399 & 0.56688 & 0.40104 & 0.40084 \\
\hline & 100 & PLF & PR & 0.012740 & 0.012804 & 0.026092 & 0.005773 & 0.005775 \\
\hline & & DIF & $\mathrm{BE}$ & 0.53997 & 0.54307 & 0.57771 & 0.40389 & 0.40384 \\
\hline & & DLF & PR & 0.023822 & 0.023825 & 0.045498 & 0.014345 & 0.014348 \\
\hline & & & $\mathrm{BE}$ & 0.51318 & 0.51296 & 0.53037 & 0.39897 & 0.39902 \\
\hline & & SELF & PR & 0.003294 & 0.003292 & 0.007042 & 0.001177 & 0.001177 \\
\hline & & & $\mathrm{BE}$ & 0.51696 & 0.51482 & 0.53228 & 0.40050 & 0.40047 \\
\hline & 200 & PLF & PR & 0.006328 & 0.006302 & 0.012761 & 0.002944 & 0.002944 \\
\hline & & & $\mathrm{BE}$ & 0.51761 & 0.51734 & 0.54254 & 0.40201 & 0.40191 \\
\hline & & DLF & PR & 0.012204 & 0.012207 & 0.023836 & 0.007338 & 0.007341 \\
\hline
\end{tabular}

Table 13: Bayes Estimates (BEs) and Posterior Risks (PRs) of 3-component Mixture of Inverse Rayleigh Distributions Using the JP under SELF, PLF and DLF with $\theta_{1}=0.50, \theta_{2}=0.50, \theta_{3}=0.50, \mathrm{p}_{1}=0.40, \mathrm{p}_{2}=0.40$ and $\mathrm{t}=15,20$

\begin{tabular}{|c|c|c|c|c|c|c|c|c|}
\hline $\mathrm{t}$ & $\mathrm{n}$ & Loss F & ons & $\begin{array}{c}\text { JP } \\
\hat{\theta}_{1}\end{array}$ & $\hat{\theta}_{2}$ & $\hat{\theta}_{3}$ & $\hat{\mathrm{p}}_{1}$ & $\hat{\mathrm{p}}_{2}$ \\
\hline \multirow{9}{*}{15} & \multirow{6}{*}{25} & \multirow{2}{*}{ SELF } & $\mathrm{BE}$ & 0.55202 & 0.55143 & 0.61820 & 0.39262 & 0.39312 \\
\hline & & & PR & 0.304288 & 0.033994 & 0.099941 & 0.008236 & 0.008240 \\
\hline & & \multirow{2}{*}{ PLF } & $\mathrm{BE}$ & 0.57768 & 0.58651 & 0.66873 & 0.40319 & 0.40335 \\
\hline & & & PR & 0.053838 & 0.054654 & 0.116811 & 0.020700 & 0.020697 \\
\hline & & \multirow{2}{*}{ DLF } & $\mathrm{BE}$ & 0.60700 & 0.60329 & 0.75817 & 0.41384 & 0.41388 \\
\hline & & & PR & 0.091001 & 0.090991 & 0.166995 & 0.050675 & 0.050666 \\
\hline & & \multirow{2}{*}{ SELF } & $\mathrm{BE}$ & 0.5207 & 0.52197 & 0.54642 & 0.39629 & 0.39626 \\
\hline & & & PR & 0.014284 & 0.014391 & 0.033356 & 0.004439 & 0.004439 \\
\hline & & \multirow{2}{*}{ PLF } & $\mathrm{BE}$ & 0.53929 & 0.53277 & 0.57225 & 0.40174 & 0.40167 \\
\hline \multirow{3}{*}{0} & 50 & & PR & 0.026033 & 0.025724 & 0.053315 & 0.011128 & 0.011130 \\
\hline & & \multirow{2}{*}{ DLF } & $\mathrm{BE}$ & 0.55118 & 0.55527 & 0.60131 & 0.40725 & 0.40754 \\
\hline & & & PR & 0.047703 & 0.047666 & 0.091037 & 0.027522 & 0.027488 \\
\hline
\end{tabular}




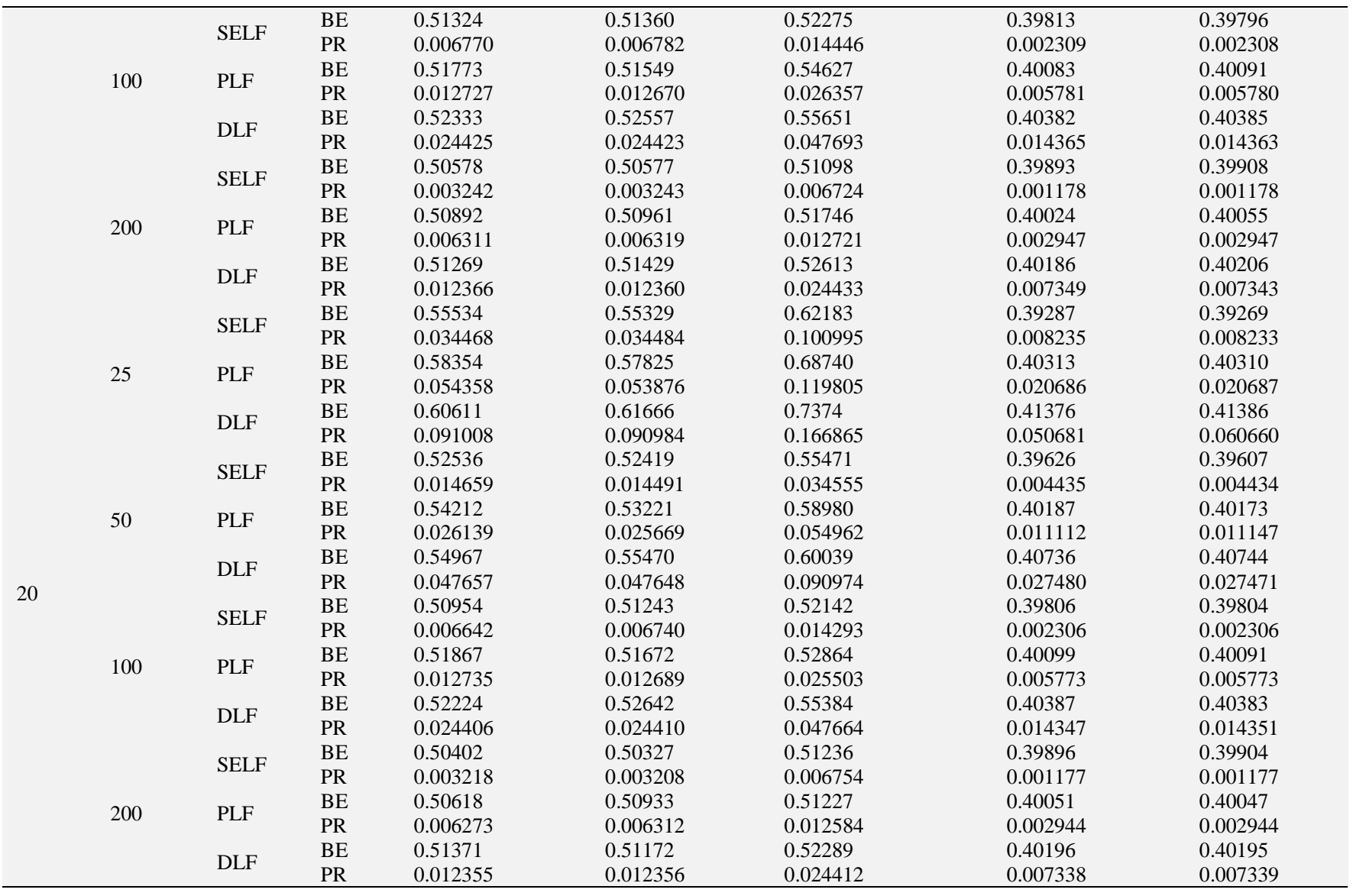

Table 14: Bayes Estimates (BEs) and Posterior Risks (PRs) of 3-component Mixture of Inverse Rayleigh Distributions Using the GP under SELF, PLF and DLF with $\theta_{1}=0.25, \theta_{2}=0.50, \theta_{3}=0.75, \mathrm{p}_{1}=0.20, \mathrm{p}_{2}=0.65$ and $\mathrm{t}=15,20$

\begin{tabular}{|c|c|c|c|c|c|c|c|c|}
\hline $\mathrm{t}$ & $\mathrm{n}$ & \multicolumn{2}{|c|}{ Loss Functions } & $\begin{array}{l}\text { GP } \\
\hat{\theta}_{1}\end{array}$ & $\hat{\theta}_{2}$ & $\hat{\theta}_{3}$ & $\hat{\mathrm{p}}_{1}$ & $\hat{\mathrm{p}}_{2}$ \\
\hline \multirow{19}{*}{15} & \multirow{6}{*}{25} & \multirow{2}{*}{ SELF } & $\mathrm{BE}$ & 0.37422 & 0.60723 & 1.40427 & 0.24916 & 0.58307 \\
\hline & & & PR & 0.031562 & 0.021855 & 0.348811 & 0.006041 & 0.007855 \\
\hline & & \multirow{2}{*}{ PLF } & $\mathrm{BE}$ & 0.39114 & 0.61177 & 1.50023 & 0.26108 & 0.58975 \\
\hline & & & PR & 0.058080 & 0.032423 & 0.193981 & 0.023685 & 0.013401 \\
\hline & & \multirow{2}{*}{ DLF } & $\mathrm{BE}$ & 0.42524 & 0.62819 & 1.63483 & 0.27317 & 0.59691 \\
\hline & & & PR & 0.143209 & 0.052262 & 0.125178 & 0.088819 & 0.022555 \\
\hline & \multirow{5}{*}{50} & SELF & PR & 0.009356 & 0.009167 & 0.120313 & 0.003136 & 0.004261 \\
\hline & & \multirow{2}{*}{ PLF } & $\mathrm{BE}$ & 0.31326 & 0.55804 & 1.11408 & 0.23359 & 0.61241 \\
\hline & & & PR & 0.026700 & 0.016007 & 0.094904 & 0.013616 & 0.006975 \\
\hline & & \multirow{2}{*}{ DLF } & $\mathrm{BE}$ & 0.32731 & 0.56343 & 1.20517 & 0.24070 & 0.61586 \\
\hline & & & PR & 0.083404 & 0.028485 & 0.083444 & 0.057429 & 0.011364 \\
\hline & \multirow{4}{*}{100} & \multirow{2}{*}{ SELF } & $\mathrm{BE}$ & 0.27417 & 0.52197 & 0.90843 & 0.21406 & 0.63326 \\
\hline & & & PR & 0.003773 & 0.004124 & 0.049299 & 0.001589 & 0.002195 \\
\hline & & PLF & $\mathrm{BE}$ & 0.28075 & 0.52459 & 0.95500 & 0.21778 & 0.63483 \\
\hline & & DLF & PR & 0.045501 & 0.014685 & 0.052786 & 0.033519 & 0.005443 \\
\hline & \multirow{6}{*}{200} & \multirow{2}{*}{ SELF } & $\mathrm{BE}$ & 0.26387 & 0.51071 & 0.84892 & 0.20718 & 0.64142 \\
\hline & & & PR & 0.001741 & 0.001991 & 0.022665 & 0.000798 & 0.001118 \\
\hline & & \multirow{2}{*}{ PLF } & $\mathrm{BE}$ & 0.26313 & 0.51320 & 0.85375 & 0.20913 & 0.64230 \\
\hline & & & PR & 0.006309 & 0.003865 & 0.025358 & 0.003835 & 0.001742 \\
\hline \multirow{13}{*}{20} & & \multirow{2}{*}{ DLF } & $\mathrm{BE}$ & 0.26914 & 0.51263 & 0.85354 & 0.21100 & 0.64324 \\
\hline & & & PR & 0.02384 & 0.007516 & 0.029477 & 0.018263 & 0.002710 \\
\hline & \multirow{6}{*}{25} & \multirow{2}{*}{ SELF } & $\mathrm{BE}$ & 0.36371 & 0.60520 & 1.4207 & 0.24906 & 0.58327 \\
\hline & & & PR & 0.028910 & 0.021631 & 0.353566 & 0.006036 & 0.007848 \\
\hline & & \multirow{2}{*}{ PLF } & $\mathrm{BE}$ & 0.39854 & 0.61388 & 1.50819 & 0.26086 & 0.58992 \\
\hline & & & PR & 0.059221 & 0.032513 & 0.194804 & 0.023677 & 0.013380 \\
\hline & & DI F & $\mathrm{BE}$ & 0.42139 & 0.63853 & 1.62286 & 0.27332 & 0.59667 \\
\hline & & DLF & PR & 0.14299 & 0.052258 & 0.125013 & 0.088669 & 0.022551 \\
\hline & & SEI F & $\mathrm{BE}$ & 0.29891 & 0.54622 & 1.08893 & 0.22681 & 0.60899 \\
\hline & & SELF & PR & 0.009092 & 0.009032 & 0.123026 & 0.003134 & 0.004257 \\
\hline & 50 & PIF & $\mathrm{BE}$ & 0.31529 & 0.55940 & 1.14757 & 0.23359 & 0.61256 \\
\hline & 100 & SEIF & $\mathrm{BE}$ & 0.27448 & 0.52211 & 0.92324 & 0.21404 & 0.63333 \\
\hline & 100 & SELF & PR & 0.003790 & 0.004128 & 0.050646 & 0.001588 & 0.002193 \\
\hline
\end{tabular}




\begin{tabular}{lllllll}
\hline & PLF & BE & 0.27966 & 0.52263 & 0.95315 & 0.63499 \\
& & PR & 0.012867 & 0.007702 & 0.050910 & 0.003459 \\
& DLF & BE & 0.28802 & 0.53025 & 0.98208 & 0.007356 \\
& & PR & 0.045476 & 0.014684 & 0.052686 & 0.22151 \\
& SELF & BE & 0.26560 & 0.51228 & 0.83382 & 0.033497 \\
& & PR & 0.001765 & 0.002003 & 0.021861 & 0.20718 \\
& & BE & 0.26611 & 0.51329 & 0.85924 & 0.000798 \\
& PLF & PR & 0.006377 & 0.003865 & 0.025489 & 0.20913 \\
& & BE & 0.26807 & 0.51419 & 0.85609 & 0.003834 \\
& DLF & PR & 0.023826 & 0.007514 & 0.029443 & 0.21102 \\
\hline
\end{tabular}

Table 15: Bayes Estimates (BEs) and Posterior Risks (PRs) of 3-component Mixture of Inverse Rayleigh Distributions Using the GP under SELF, PLF and DLF with $\theta_{1}=0.75, \theta_{2}=0.50, \theta_{3}=0.25, \mathrm{p}_{1}=0.65, \mathrm{p}_{2}=0.20$ and $\mathrm{t}=15,20$

\begin{tabular}{|c|c|c|c|c|c|c|c|c|}
\hline $\mathrm{t}$ & $\mathrm{n}$ & Loss Functions & & $\begin{array}{l}\text { GP } \\
\hat{\theta}_{1}\end{array}$ & $\hat{\theta}_{2}$ & $\hat{\theta}_{3}$ & $\hat{\mathrm{p}}_{1}$ & $\hat{\mathrm{p}}_{2}$ \\
\hline \multirow{17}{*}{15} & \multirow{6}{*}{25} & \multirow{2}{*}{ SELF } & $\mathrm{BE}$ & 0.82927 & 0.85261 & 0.55109 & 0.61556 & 0.21679 \\
\hline & & & PR & 0.043317 & 0.128387 & 0.058983 & 0.007650 & 0.005490 \\
\hline & & \multirow{2}{*}{ PLF } & $\mathrm{BE}$ & 0.86677 & 0.90082 & 0.58593 & 0.62168 & 0.22907 \\
\hline & & & PR & 0.048914 & 0.114347 & 0.075699 & 0.012366 & 0.024618 \\
\hline & & \multirow{2}{*}{ DLF } & $\mathrm{BE}$ & 0.88344 & 0.99082 & 0.62287 & 0.62794 & 0.24218 \\
\hline & & & $\mathrm{PR}$ & 0.055646 & 0.122907 & 0.125097 & 0.019803 & 0.104616 \\
\hline & \multirow{5}{*}{50} & SELF & PR & 0.020189 & 0.041850 & 0.019987 & 0.004192 & 0.003050 \\
\hline & & \multirow{2}{*}{ PLF } & $\mathrm{BE}$ & 0.82296 & 0.70081 & 0.42421 & 0.64297 & 0.21985 \\
\hline & & & PR & 0.024038 & 0.054215 & 0.039479 & 0.006535 & 0.014099 \\
\hline & & \multirow{2}{*}{ DLF } & $\mathrm{BE}$ & 0.82619 & 0.72671 & 0.45190 & 0.64627 & 0.22710 \\
\hline & & & PR & 0.029006 & 0.075950 & 0.090939 & 0.010139 & 0.063139 \\
\hline & \multirow{4}{*}{100} & \multirow{2}{*}{ SELF } & $\mathrm{BE}$ & 0.76898 & 0.57705 & 0.31313 & 0.64245 & 0.20487 \\
\hline & & & PR & 0.009131 & 0.015844 & 0.005828 & 0.002172 & 0.001541 \\
\hline & & PLF & $\mathrm{BE}$ & 0.77271 & 0.58619 & 0.32796 & 0.64420 & 0.20853 \\
\hline & & DLF & PR & 0.014940 & 0.043272 & 0.052668 & 0.005233 & 0.035415 \\
\hline & & \multirow{2}{*}{ SELF } & $\mathrm{BE}$ & 0.76255 & 0.54010 & 0.28430 & 0.64604 & 0.20250 \\
\hline & & & PR & 0.004479 & 0.007109 & 0.002533 & 0.001194 & 0.000793 \\
\hline \multirow{26}{*}{20} & & \multirow{2}{*}{ PLF } & $\mathrm{BE}$ & 0.75876 & 0.55233 & 0.28834 & 0.64706 & 0.20443 \\
\hline & 200 & & PR & 0.005764 & 0.012897 & 0.008555 & 0.001719 & 0.003862 \\
\hline & & \multirow{2}{*}{ DLF } & $\mathrm{BE}$ & 0.76583 & 0.55220 & 0.29357 & 0.64807 & 0.20632 \\
\hline & & & PR & 0.007581 & 0.023218 & 0.029450 & 0.002874 & 0.018880 \\
\hline & & \multirow{2}{*}{ SELF } & $\mathrm{BE}$ & 0.83230 & 0.87476 & 0.53722 & 0.61567 & 0.21677 \\
\hline & & & PR & 0.043653 & 0.137201 & 0.056175 & 0.007643 & 0.005485 \\
\hline & & \multirow{2}{*}{ PLF } & $\mathrm{BE}$ & 0.86782 & 0.91090 & 0.55457 & 0.62168 & 0.22913 \\
\hline & 25 & & PR & 0.048958 & 0.115548 & 0.071635 & 0.012357 & 0.024603 \\
\hline & & \multirow{2}{*}{ DLF } & $\mathrm{BE}$ & 0.88076 & 0.94978 & 0.61573 & 0.62803 & 0.24208 \\
\hline & & & PR & 0.055606 & 0.122815 & 0.125043 & 0.019767 & 0.104502 \\
\hline & & & $\mathrm{BE}$ & 0.81359 & 0.67782 & 0.40399 & 0.63965 & 0.21279 \\
\hline & & SELF & PR & 0.020373 & 0.042506 & 0.019598 & 0.004186 & 0.003046 \\
\hline & & & $\mathrm{BE}$ & 0.81672 & 0.70551 & 0.41586 & 0.64299 & 0.21984 \\
\hline & 50 & PLF & PR & 0.023848 & 0.054547 & 0.038681 & 0.006528 & 0.014088 \\
\hline & & & $\mathrm{BE}$ & 0.76954 & 0.57767 & 0.31850 & 0.64254 & 0.20482 \\
\hline & & SELF & PR & 0.009119 & 0.015944 & 0.006063 & 0.002169 & 0.001538 \\
\hline & & & $\mathrm{BE}$ & 0.77946 & 0.58893 & 0.32588 & 0.64430 & 0.20847 \\
\hline & 100 & PLF & PR & 0.011684 & 0.025756 & 0.017395 & 0.003372 & 0.007446 \\
\hline & & & $\mathrm{BE}$ & 0.77680 & 0.60440 & 0.33650 & 0.64597 & 0.21227 \\
\hline & & DLF & PR & 0.014933 & 0.043251 & 0.052658 & 0.005226 & 0.035393 \\
\hline & & SEI F & $\mathrm{BE}$ & 0.76233 & 0.53906 & 0.28019 & 0.64618 & 0.20244 \\
\hline & & SELF & PR & 0.004475 & 0.007068 & 0.002459 & 0.001111 & 0.000785 \\
\hline & 200 & & $\mathrm{BE}$ & 0.76201 & 0.54534 & 0.28941 & 0.64703 & 0.20438 \\
\hline & 200 & PLF & PR & 0.005787 & 0.012728 & 0.008579 & 0.001716 & 0.003858 \\
\hline & & & $\mathrm{BE}$ & 0.76821 & 0.54953 & 0.28884 & 0.64810 & 0.20634 \\
\hline & & DLF & PR & 0.007580 & 0.023203 & 0.029429 & 0.003042 & 0.018948 \\
\hline
\end{tabular}

Table 16: Bayes Estimates (BEs) and Posterior Risks (PRs) of 3-component Mixture of Inverse Rayleigh Distributions Using the GP under SELF, PLF and DLF with $\theta_{1}=0.50, \theta_{2}=0.50, \theta_{3}=0.50, \mathrm{p}_{1}=0.40, \mathrm{p}_{2}=0.40$ and $\mathrm{t}=15,20$

\begin{tabular}{|c|c|c|c|c|c|c|c|c|}
\hline $\mathrm{t}$ & $\mathrm{n}$ & Loss Functions & & $\begin{array}{c}\text { JP } \\
\hat{\theta}_{1}\end{array}$ & $\hat{\theta}_{2}$ & $\hat{\theta}_{3}$ & $\hat{\mathrm{p}}_{1}$ & $\hat{\mathrm{p}}_{2}$ \\
\hline \multirow{8}{*}{15} & \multirow{6}{*}{25} & \multirow{2}{*}{ SELF } & $\mathrm{BE}$ & 0.59691 & 0.66389 & 0.88846 & 0.41582 & 0.38306 \\
\hline & & & PR & 0.036413 & 0.040213 & 0.118906 & 0.007847 & 0.007634 \\
\hline & & \multirow{2}{*}{ PLF } & $\mathrm{BE}$ & 0.62452 & 0.68879 & 0.95756 & 0.42524 & 0.39301 \\
\hline & & & PR & 0.053247 & 0.053525 & 0.109718 & 0.018668 & 0.019684 \\
\hline & & \multirow{2}{*}{ DLF } & $\mathrm{BE}$ & 0.65209 & 0.71000 & 1.02008 & 0.43478 & 0.40319 \\
\hline & & & PR & 0.083426 & 0.076165 & 0.11128 & 0.043420 & 0.049439 \\
\hline & \multirow{2}{*}{50} & \multirow{2}{*}{ SELF } & $\mathrm{BE}$ & 0.55224 & 0.57438 & 0.69741 & 0.40844 & 0.39101 \\
\hline & & & PR & 0.01542 & 0.015714 & 0.041181 & 0.004322 & 0.004260 \\
\hline
\end{tabular}




\begin{tabular}{|c|c|c|c|c|c|c|c|c|}
\hline & & & $\mathrm{BE}$ & 0.55567 & 0.59775 & 0.72718 & 0.41385 & 0.39626 \\
\hline & & PLF & PR & 0.025586 & 0.026147 & 0.052968 & 0.010514 & 0.010825 \\
\hline & & & $\mathrm{BE}$ & 0.57452 & 0.6057 & 0.73432 & 0.41932 & 0.40182 \\
\hline & & DLF & PR & 0.045503 & 0.043256 & 0.071580 & 0.025232 & 0.027125 \\
\hline & & SFI F & $\mathrm{BE}$ & 0.52408 & 0.53393 & 0.59090 & 0.40443 & 0.39520 \\
\hline & & SELF & PR & 0.006894 & 0.006952 & 0.015919 & 0.002277 & 0.002259 \\
\hline & & & $\mathrm{BE}$ & 0.52885 & 0.53772 & 0.60945 & 0.40730 & 0.39807 \\
\hline & 100 & PLF & PR & 0.012683 & 0.012551 & 0.025697 & 0.005609 & 0.005696 \\
\hline & & DIF & $\mathrm{BE}$ & 0.53497 & 0.55109 & 0.61497 & 0.41015 & 0.40091 \\
\hline & & & $\mathrm{BE}$ & 0.51150 & 0.51996 & 0.54766 & 0.40217 & 0.39752 \\
\hline & & SELF & PR & 0.003280 & 0.003336 & 0.007156 & 0.001227 & 0.001221 \\
\hline & 200 & & $\mathrm{BE}$ & 0.51437 & 0.52515 & 0.55177 & 0.40376 & 0.39903 \\
\hline & 200 & $\mathrm{PLF}$ & PR & 0.006301 & 0.006344 & 0.012634 & 0.002955 & 0.002977 \\
\hline & & $\mathrm{DI}$ & $\mathrm{BE}$ & 0.51586 & 0.52453 & 0.56354 & 0.40528 & 0.40040 \\
\hline & & DLF & PR & 0.012211 & 0.012047 & 0.022755 & 0.007323 & 0.007469 \\
\hline & & & $\mathrm{BE}$ & 0.59363 & 0.66657 & 0.87214 & 0.41563 & 0.38325 \\
\hline & & SELF & PR & 0.035591 & 0.040578 & 0.116424 & 0.007842 & 0.007632 \\
\hline & 25 & PLF & PR & 0.053693 & 0.054258 & 0.110251 & 0.018650 & 0.019660 \\
\hline & & & $\mathrm{BE}$ & 0.65012 & 0.73387 & 1.0077 & 0.43465 & 0.40322 \\
\hline & & DLF & PR & 0.083419 & 0.076133 & 0.111207 & 0.043415 & 0.049403 \\
\hline & & & $\mathrm{BE}$ & 0.54177 & 0.57817 & 0.68669 & 0.40854 & 0.39093 \\
\hline & & SELF & PR & 0.014743 & 0.015925 & 0.040049 & 0.004302 & 0.004257 \\
\hline & & & $\mathrm{BE}$ & 0.57065 & 0.58725 & 0.71168 & 0.41382 & 0.39641 \\
\hline & 50 & PLF & PR & 0.026262 & 0.025659 & 0.051830 & 0.010504 & 0.010810 \\
\hline & & & $\mathrm{BE}$ & 0.57144 & 0.60592 & 0.74213 & 0.41913 & 0.40181 \\
\hline 20 & & DLF & PR & 0.045491 & 0.043227 & 0.071468 & 0.025222 & 0.027094 \\
\hline 20 & & SEI & $\mathrm{BE}$ & 0.51785 & 0.53517 & 0.59717 & 0.40457 & 0.39515 \\
\hline & & SELF & PR & 0.006709 & 0.006995 & 0.016428 & 0.002275 & 0.002258 \\
\hline & & & $\mathrm{BE}$ & 0.52183 & 0.54576 & 0.60954 & 0.40730 & 0.39806 \\
\hline & 100 & PLF & PR & 0.022509 & 0.012732 & 0.025690 & 0.005605 & 0.005692 \\
\hline & & & $\mathrm{BE}$ & 0.53445 & 0.54983 & 0.61245 & 0.41012 & 0.40101 \\
\hline & & DLF & PR & 0.023828 & 0.023195 & 0.041695 & 0.013776 & 0.014549 \\
\hline & & SEI F & $\mathrm{BE}$ & 0.50891 & 0.52149 & 0.55130 & 0.40232 & 0.39754 \\
\hline & & SELF & PR & 0.003238 & 0.003355 & 0.007263 & 0.001168 & 0.001163 \\
\hline & & & $\mathrm{BE}$ & 0.51679 & 0.52561 & 0.56103 & 0.40522 & 0.40051 \\
\hline & & DLF & PR & 0.012204 & 0.012035 & 0.022753 & 0.007167 & 0.007309 \\
\hline
\end{tabular}

Table 17: Bayes Estimates (BEs) and Posterior Risks (PRs) of 3-component Mixture of Inverse Rayleigh Distributions Using the EP under SELF, PLF and DLF with $\theta_{1}=0.25, \theta_{2}=0.50, \theta_{3}=0.75, \mathrm{p}_{1}=0.20, \mathrm{p}_{2}=0.65$ and $\mathrm{t}=15,20$

\begin{tabular}{|c|c|c|c|c|c|c|c|c|}
\hline $\mathrm{t}$ & $\mathrm{n}$ & Loss Functions & & $\begin{array}{l}\mathrm{EP} \\
\hat{\theta}_{1}\end{array}$ & $\hat{\theta}_{2}$ & $\hat{\theta}_{3}$ & $\hat{\mathrm{p}}_{1}$ & $\hat{\mathrm{p}}_{2}$ \\
\hline \multirow{22}{*}{15} & \multirow{6}{*}{25} & \multirow{2}{*}{ SELF } & $\mathrm{BE}$ & 0.30920 & 0.53506 & 0.94410 & 0.22393 & 0.61115 \\
\hline & & & PR & 0.018811 & 0.017950 & 0.215536 & 0.006144 & 0.008407 \\
\hline & & \multirow{2}{*}{ PLF } & $\mathrm{BE}$ & 0.33646 & 0.54228 & 1.02548 & 0.23731 & 0.61804 \\
\hline & & & PR & 0.049928 & 0.030586 & 0.179001 & 0.026637 & 0.013675 \\
\hline & & \multirow{2}{*}{ DLF } & $\mathrm{BE}$ & 0.35373 & 0.56103 & 1.14205 & 0.25149 & 0.62497 \\
\hline & & & PR & 0.142908 & 0.055614 & 0.167032 & 0.109087 & 0.022015 \\
\hline & \multirow{6}{*}{50} & \multirow{2}{*}{ SELF } & $\mathrm{BE}$ & 0.27944 & 0.51824 & 0.86138 & 0.21248 & 0.62504 \\
\hline & & & PR & 0.007833 & 0.008386 & 0.092381 & 0.003142 & 0.004404 \\
\hline & & \multirow{2}{*}{ PLF } & $\mathrm{BE}$ & 0.29122 & 0.52165 & 0.91900 & 0.21980 & 0.62857 \\
\hline & & & PR & 0.024814 & 0.015467 & 0.094515 & 0.014536 & 0.007025 \\
\hline & & \multirow{2}{*}{ DLF } & $\mathrm{BE}$ & 0.30798 & 0.53791 & 0.95542 & 0.22734 & 0.63191 \\
\hline & & & PR & 0.083403 & 0.029445 & 0.100168 & 0.065058 & 0.011160 \\
\hline & \multirow{6}{*}{100} & \multirow{2}{*}{ SELF } & $\mathrm{BE}$ & 0.26453 & 0.50554 & 0.80709 & 0.20643 & 0.64205 \\
\hline & & & PR & 0.003496 & 0.003936 & 0.043761 & 0.001588 & 0.002229 \\
\hline & & \multirow{2}{*}{ PLF } & $\mathrm{BE}$ & 0.26945 & 0.51066 & 0.83838 & 0.21018 & 0.64393 \\
\hline & & & PR & 0.012403 & 0.007655 & 0.050203 & 0.007620 & 0.003466 \\
\hline & & \multirow{2}{*}{ DLF } & $\mathrm{BE}$ & 0.27792 & 0.51443 & 0.85020 & 0.21409 & 0.64554 \\
\hline & & & PR & 0.045494 & 0.014937 & 0.058942 & 0.035921 & 0.005377 \\
\hline & \multirow{6}{*}{200} & \multirow{2}{*}{ SELF } & $\mathrm{BE}$ & 0.25762 & 0.50567 & 0.77852 & 0.20323 & 0.64595 \\
\hline & & & PR & 0.001660 & 0.001968 & 0.020278 & 0.0008000 & 0.001156 \\
\hline & & \multirow{2}{*}{ PLF } & $\mathrm{BE}$ & 0.26188 & 0.50663 & 0.79073 & 0.20520 & 0.64686 \\
\hline & & & PR & 0.006279 & 0.003849 & 0.024970 & 0.003905 & 0.001744 \\
\hline \multirow{12}{*}{20} & & \multirow{2}{*}{ DLF } & $\mathrm{BE}$ & 0.26499 & 0.50673 & 0.80782 & 0.20718 & 0.6478 \\
\hline & & & PR & 0.023828 & 0.007581 & 0.031331 & 0.019018 & 0.002855 \\
\hline & \multirow{6}{*}{25} & \multirow{2}{*}{ SELF } & $\mathrm{BE}$ & 0.30068 & 0.52915 & 0.92535 & 0.22403 & 0.61126 \\
\hline & & & PR & 0.018110 & 0.017517 & 0.20643 & 0.006142 & 0.008398 \\
\hline & & \multirow[b]{2}{*}{ PLF } & $\mathrm{BE}$ & 0.32422 & 0.54462 & 1.05716 & 0.23736 & 0.61807 \\
\hline & & & PR & 0.048095 & 0.030704 & 0.184347 & 0.026624 & 0.013663 \\
\hline & & \multirow{2}{*}{ DLF } & $\mathrm{BE}$ & 0.35530 & 0.56231 & 1.15575 & 0.25141 & 0.62502 \\
\hline & & & PR & 0.142893 & 0.055587 & 0.166851 & 0.109071 & 0.021987 \\
\hline & \multirow{4}{*}{50} & \multirow{2}{*}{ SELF } & $\mathrm{BE}$ & 0.28290 & 0.51573 & 0.85127 & 0.21259 & 0.62505 \\
\hline & & & PR & 0.008015 & 0.008310 & 0.092115 & 0.003142 & 0.004400 \\
\hline & & \multirow{2}{*}{ PLF } & $\mathrm{BE}$ & 0.29107 & 0.52410 & 0.91085 & 0.21981 & 0.62848 \\
\hline & & & PR & 0.024789 & 0.015537 & 0.093592 & 0.014529 & 0.007021 \\
\hline
\end{tabular}




\begin{tabular}{|c|c|c|c|c|c|c|c|}
\hline & \multirow{2}{*}{ DLF } & $\mathrm{BE}$ & 0.30182 & 0.53129 & 0.94785 & 0.22734 & 0.63192 \\
\hline & & PR & 0.08335 & 0.029432 & 0.100063 & 0.065005 & 0.011146 \\
\hline \multirow{6}{*}{100} & \multirow{2}{*}{ SELF } & $\mathrm{BE}$ & 0.26483 & 0.50942 & 0.80936 & 0.20640 & 0.64207 \\
\hline & & PR & 0.003506 & 0.003996 & 0.044051 & 0.001586 & 0.002227 \\
\hline & \multirow{2}{*}{ PLF } & $\mathrm{BE}$ & 0.27277 & 0.51334 & 0.82514 & 0.21024 & 0.64376 \\
\hline & & PR & 0.012548 & 0.007696 & 0.049343 & 0.007617 & 0.003465 \\
\hline & \multirow{2}{*}{ DLF } & $\mathrm{BE}$ & 0.27916 & 0.51736 & 0.87226 & 0.21404 & 0.64551 \\
\hline & & PR & 0.045494 & 0.014935 & 0.058873 & 0.035920 & 0.005375 \\
\hline \multirow{5}{*}{200} & SELF & $\mathrm{BE}$ & 0.25557 & 0.50155 & 0.77322 & 0.20321 & 0.64602 \\
\hline & \multirow{2}{*}{ PLF } & $\mathrm{BE}$ & 0.25803 & 0.50727 & 0.79692 & 0.20519 & 0.64684 \\
\hline & & PR & 0.006184 & 0.003852 & 0.025128 & 0.003903 & 0.001742 \\
\hline & \multirow{2}{*}{ DLF } & $\mathrm{BE}$ & 0.26682 & 0.50702 & 0.79592 & 0.20715 & 0.64773 \\
\hline & & PR & 0.023824 & 0.007579 & 0.03129 & 0.018932 & 0.002692 \\
\hline
\end{tabular}

Table 18: Bayes Estimates (BEs) and Posterior Risks (PRs) of 3-component Mixture of Inverse Rayleigh Distributions Using the EP under SELF, PLF and DLF with $\theta_{1}=0.75, \theta_{2}=0.50, \theta_{3}=0.25, \mathrm{p}_{1}=0.65, \mathrm{p}_{2}=0.20$ and $\mathrm{t}=15,20$

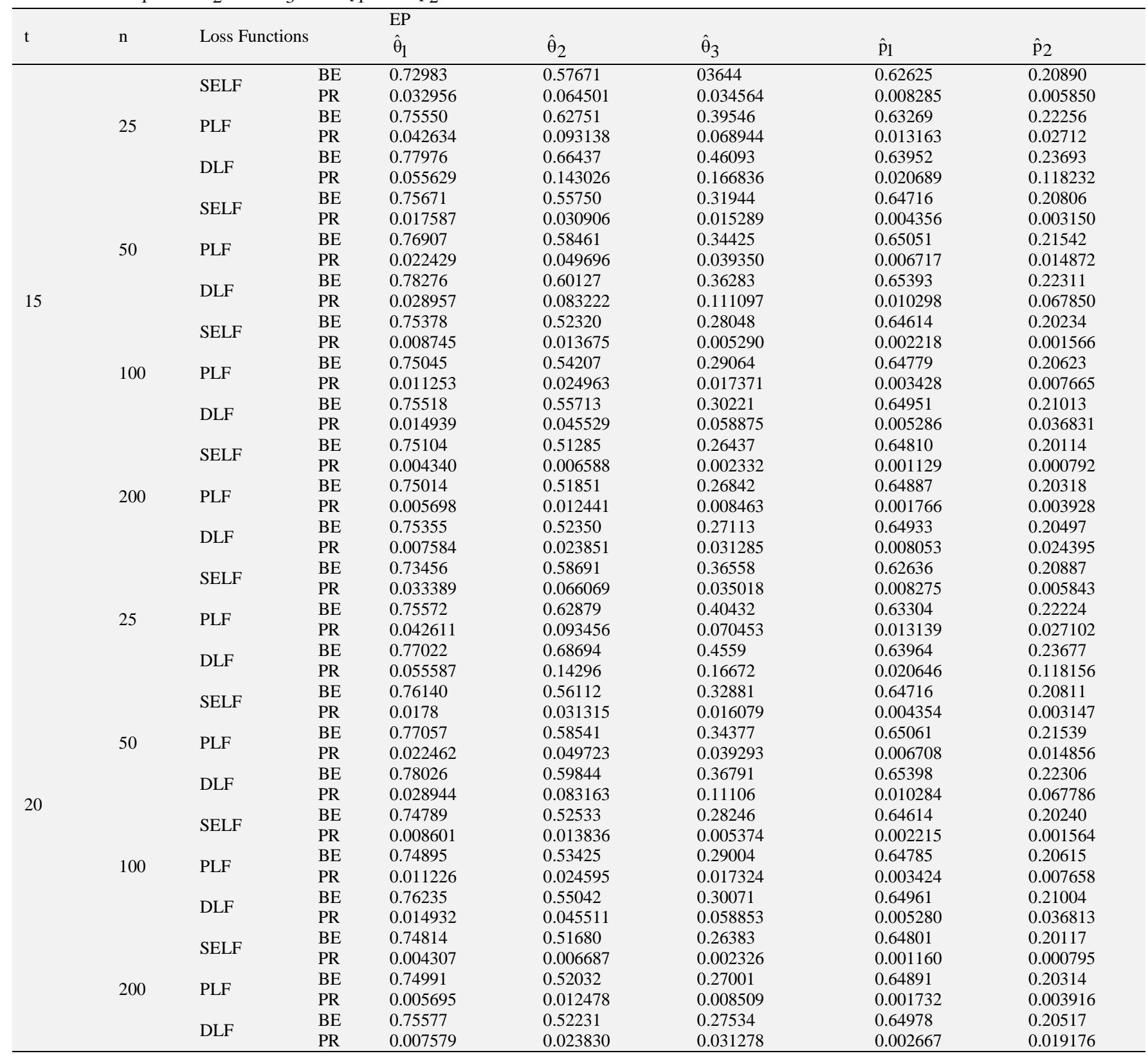

Table 19: Bayes Estimates (BEs) and Posterior Risks (PRs) of 3-component Mixture of Inverse Rayleigh Distributions Using the EP under SELF, PLF and DLF with $\theta_{1}=0.50, \theta_{2}=0.50, \theta_{3}=0.50, \mathrm{p}_{1}=0.40, \mathrm{p}_{2}=0.40$ and $\mathrm{t}=15,20$

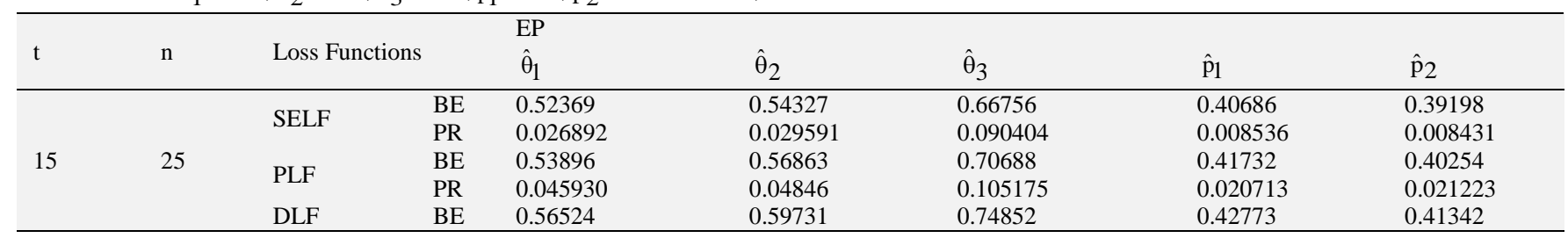




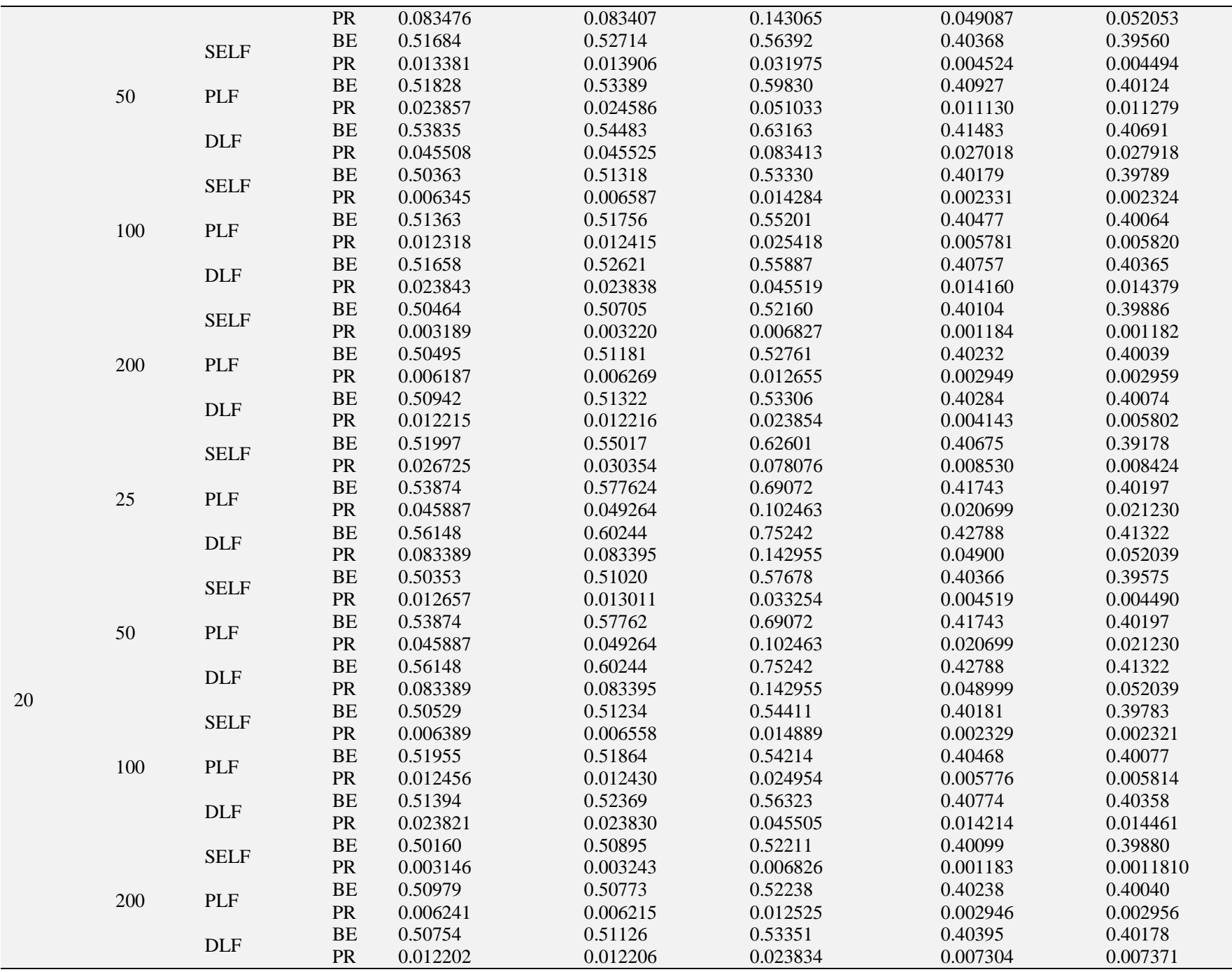

\section{Conclusion}

In this study, we have considered the Bayesian analysis of 3component mixture of inverse Rayleigh distributions using the non-informative (Uniform and Jeffreys') and the informative (Gamma and Exponential) priors under SELF, PLF and DLF. The purpose of this paper is to find out the appropriate combinations of prior distributions and loss functions to estimate the parameters of the 3-component mixture of the inverse Rayleigh distributions. We conducted a comprehensive simulation study to determine the relative performance of the Bayes estimators. From simulated results, we observed that an increase in the sample size and test termination time provides better Bayes estimators. Furthermore, as sample size increases (decreases) the posterior risks of Bayes estimator's decreases (increase) for a fixed test termination time. Also, the DLF is observed as a suitable choice for estimating component parameters and SELF is preferable for estimating the proportion parameters. Finally, we conclude that the GP is suitable prior in order to estimate the component parameters. When SELF is used, the GP is an appropriate prior for proportion parameters. The same pattern is observed for the JP when non-informative priors are considered.

\section{References}

[1] Abdel-Monem, A. (2003). Estimation and Prediction for the Inverse Rayleigh Life Distribution, M. Sc. Thesis Faculty of Education, Ain Shames University.

[2] Ali, S. (2015). "Mixture of the inverse Rayleigh distribution: Properties and estimation in a Bayesian framework." Applied Mathe- matical

Modelling $39(2):$

$515-530$. http://dx.doi.org/10.1016/j.apm.2014.05.039.

[3] Aslam, M. (2003). "An application of prior predictive distribution to elicit the prior density." Journal of Statistical Theory and applications 2(1): 70-83

[4] Aslam, M., M. Tahir, et al. (2015). "A 3-Component Mixture of Rayleigh Distributions: Properties and Estimation in Bayesian Framework."

[5] Berger, J. (1985). "Statistical decision theory and Bayesian analysis."

[6] DeGroot, M. H. (2005). Optimal statistical decisions, John Wiley \& Sons.

[7] Dey, S. (2012). "Bayesian estimation of the parameter and reliability function of an inverse Rayleigh distribution." Malaysian Journal of Mathematical Sciences 6(1): 113-124.

[8] Gharraph, M. (1993). "Comparison of estimators of location measures of an inverse Rayleigh distribution." The Egyptian Statistical Journal 37: 295-309.

[9] Gijbels, I. (2010). "Censored data." Wiley Interdisciplinary Reviews: Computational Statistics 2(2): 178-188. http://dx.doi.org/10.1002/wics.80.

[10] Howlader, H. A., A. M. Hossain, et al. (2009). "Bayesian prediction bounds for Rayleigh and inverse Rayleigh lifetime models." Journal of Applied Statistical Science 17(1): 131.

[11] Jeffreys, H. (1946). An invariant form for the prior probability in estimation problems. Proceedings of the Royal Society of London a: Mathematical, Physical and Engineering Sciences, the Royal Society. http://dx.doi.org/10.1098/rspa.1946.0056.

[12] Jeffreys, H. (1961). Theory of probability, Clarendon Press, Oxford

[13] Kalbfleisch, J. D. and R. L. Prentice (2011). The statistical analysis of failure time data, John Wiley \& Sons.

[14] Kazmi, S., M. Aslam, et al. (2012). "On the Bayesian estimation for two component mixture of Maxwell distribution, assuming type I censored data." SOURCE International Journal of Applied Science $\&$ Technology 2 . 
[15] Legendre, A. M. (1805). Nouvelles méthodes pour la détermination des orbites des comètes, F. Didot.

[16] Mendenhall, W. and R. Hader (1958). "Estimation of parameters of mixed exponentially distributed failure time distributions from censored life test data." Biometrika 45(3-4): 504-520. http://dx.doi.org/10.1093/biomet/45.3-4.504

[17] Noor, F. and M. Aslam (2013). "Bayesian inference of the inverse weibull mixture distribution using Type-I censoring." Journal of $\begin{array}{llll}\text { Applied } & \text { Statistics } & \text { 40(5): } & \end{array}$ http://dx.doi.org/10.1080/02664763.2013.780157.

[18] Norstrøm, J. G. (1996). "The use of precautionary loss functions in risk analysis." Reliability, IEEE Transactions on 45(3): 400-403. http://dx.doi.org/10.1109/24.536992.

[19] Soliman, A. A. and F. M. Al-Aboud (2008). "Bayesian inference using record values from Rayleigh model with application." European Journal of Operational Research 185(2): 659-672. http://dx.doi.org/10.1016/j.ejor.2007.01.023.

[20] Soliman, A., E. A. Amin, et al. (2010). "Estimation and prediction from inverse Rayleigh distribution based on lower record values." Applied Mathematical Sciences 4(62): 3057-3066.

[21] Sultan, K., M. Ismail, et al. (2007). "Mixture of two inverse weibull distributions: Properties and estimation." Computational Statistics \& Data Analysis 51(11): 5377-5387. http://dx.doi.org/10.1016/j.csda.2006.09.016.

[22] Voda, V. G. (1972). "On the inverse Rayleigh distributed random variable." Rep. Statist. App. Res., JUSE 19: 13-21. 